\title{
Takens-Bogdanov bifurcation of travelling-wave solutions in pipe flow
}

\author{
F. MELLIBOVSKY ${ }^{1} \dagger$ AND B. ECKHARDT ${ }^{2,3}$ \\ ${ }^{1}$ Departament de Física Aplicada, Universitat Politècnica de Catalunya, 08034 Barcelona, Spain \\ ${ }^{2}$ Fachbereich Physik, Philipps-Universität Marburg, D-35032 Marburg, Germany \\ ${ }^{3}$ Laboratory for Aero and Hydrodynamics, Delft University of Technology, \\ 2628 CA Delft, The Netherlands
}

(Received 1 February 2010; revised 29 September 2010; accepted 1 October 2010;

first published online 25 January 2011)

The appearance of travelling-wave-type solutions in pipe Poiseuille flow that are disconnected from the basic parabolic profile is numerically studied in detail. We focus on solutions in the twofold azimuthally-periodic subspace because of their special stability properties, but relate our findings to other solutions as well. Using timestepping, an adapted Krylov-Newton method and Arnoldi iteration for the computation and stability analysis of relative equilibria, and a robust pseudo-arclength continuation scheme, we unfold a double-zero (Takens-Bogdanov) bifurcating scenario as a function of Reynolds number $(R e)$ and wavenumber $(\kappa)$. This scenario is extended, by the inclusion of higher-order terms in the normal form, to account for the appearance of supercritical modulated waves emanating from the upper branch of solutions at a degenerate Hopf bifurcation. We provide evidence that these modulated waves undergo a fold-of-cycles and compute some solutions on the unstable branch. These waves are shown to disappear in saddle-loop bifurcations upon collision with lowerbranch solutions, in accordance with the bifurcation scenario proposed. The travellingwave upper-branch solutions are stable within the subspace of twofold periodic flows, and their subsequent secondary bifurcations could contribute to the formation of the phase space structures that are required for turbulent dynamics at higher Re.

Key words: bifurcation, transition to turbulence

\section{Introduction}

Subcritical transition and the sustainment of turbulence at moderate flow rates in shear flows is a problem of great theoretical complexity and practical relevance (Grossmann 2000; Eckhardt et al. 2007; Eckhardt 2009). Since Osborne Reynolds' (1883) much celebrated work on the onset of turbulent motion in a straight pipe of circular cross-section, this problem has become a benchmark of transition without linear instability, i.e. subcritical transition. Many theoretical (Boberg \& Brosa 1988; Brosa \& Grossmann 1999), numerical (Schmid \& Henningson 1994; Zikanov 1996; Shan et al. 1999) and experimental (Wygnanski \& Champagne 1973; Wygnanski, Sokolov \& Friedman 1975; Darbyshire \& Mullin 1995; Hof et al. 2006) efforts have been devoted to comprehending how the laminar state (Hagen-Poiseuille 
flow) becomes unstable to finite-amplitude perturbations despite its linear stability (Pfenniger 1961; Meseguer \& Trefethen 2003).

The numerical computation of finite-amplitude solutions in the form of travelling waves (Faisst \& Eckhardt 2003; Wedin \& Kerswell 2004; Eckhardt et al. 2008), whose existence relies on a mechanism called the self-sustained process (Waleffe 1997), initially advanced to account for turbulent regeneration, has brought renewed interest to the problem within the dynamical systems community. These waves, which are disconnected from the basic flow, typically appear in saddle-node bifurcations and break some of the symmetries of the problem while retaining others (Pringle \& Kerswell 2007; Pringle, Duguet \& Kerswell 2009). Their lower branches have been repeatedly shown to dwell at the critical threshold (Duguet, Willis \& Kerswell 2008b; Mellibovsky \& Meseguer 2009) separating the basins of attraction of laminar and turbulent flows, which suggests their implication in the transition process. Upperbranch solutions, with wall frictions closer to turbulence, seem to play a role in developed turbulence (Kerswell \& Tutty 2007; Schneider, Eckhardt \& Vollmer 2007a) and have been observed in experiments (Hof et al. 2004). Stability analysis of pipe flow travelling waves has recently led to the computation of a branch of modulated waves emerging from a Hopf bifurcation (Duguet, Pringle \& Kerswell 2008a). Their long period and rapid destabilization renders the branch difficult to continue with standard methods that seem to work well in other shear flows such as plane Couette (Viswanath 2007).

The twofold discrete azimuthally-periodic subspace constitutes a special case in that it contains a family of travelling waves with unusually low-dimensional unstable manifolds. Most known families of travelling waves exhibit at least one unstable mode within their subspace that is shared by all waves, thereby rendering the family globally unstable. This is not the case of a specific shift-reflect, twofold azimuthally-periodic family of travelling waves, the special stability properties of which can be qualified as convenient. Lower-branch solutions of this family possess a single unstable direction within the subspace, which ultimately assists bare symmetryrestricted time evolution in converging them numerically (Duguet et al. 2008b), when adequately combined with edge-tracking techniques (Skufca, Yorke \& Eckhardt 2006; Schneider, Eckhardt \& Yorke 2007b). The lower branch being a saddle, it would not be surprising if the upper branch constituted a stable node, at least for some of the parameter values. Additional unstable directions arise when analysing the stability in full space or when considering subharmonic instability to longer wavelengths, but the convenient stability properties within their azimuthal subspace, which can by itself sustain turbulence, renders it a well accessible system to study the various bifurcations and transitions that can occur. Many of them can be expected to occur also in the full, not symmetry-reduced space, but they would technically be more difficult to analyse.

The current analysis focuses on a symmetry-reduced family of travelling waves in circular pipe flow, but we expect that the results, in particular the occurrence of Takens-Bogdanov bifurcations, albeit in a context of global instability, are a common feature and can be expected in a vast range of other travelling-wave families in pipe flow and several equilibria and relative equilibria in a variety of other shear flows such as plane Couette (Nagata 1997; Wang, Gibson \& Waleffe 2007) or plane Poiseuille (Pugh \& Saffman 1988; Ehrenstein \& Koch 1991; Soibelman \& Meiron 1991) flows.

As a result of the findings reported in this study, modulated waves arising from early Hopf bifurcations off upper-branch travelling waves are not necessarily good candidates for subsequent destabilization at higher $R e$ that could lead to chaotic 
dynamics, at least not those emerging in the broader context of Takens-Bogdanov bifurcations, as they are shown to disappear uneventfully.

The outline of the paper is as follows. In $\S 2$, we present the pipe Poiseuille flow problem and sketch a numerical scheme for the integration of the resulting equations. The symmetries of the problem are discussed, and numerical methods for the computation and stability analysis of relative equilibria are introduced. Some aspects of normal form reduction in the case of bifurcating relative equilibria are also reviewed in $\S 2$. The main results of the computations are presented in $\S 3$, where the different types of solutions resulting from an extensive parameter space exploration are exhibited, and evidence of a double-zero bifurcation scenario is exposed. These results are thoroughly discussed and explained in $\S 4$ by considering the inclusion of higher-order terms in the relevant normal form. Conclusions and ensuing prospects and challenges are summarized in $\S 5$.

\section{Formulation and methods}

\subsection{Equations and numerical scheme}

The constant flow-rate motion of an incompressible Newtonian fluid through a straight pipe of circular cross-section is considered. The governing parameter is the Reynolds number, defined as $R e=U D / \nu$, where $U$ is the mean axial flow speed, $D$ is the pipe diameter and $v$ is the kinematic viscosity of the fluid. The dynamics is governed by the incompressible Navier-Stokes equations.

In cylindrical non-dimensional coordinates $\boldsymbol{x}=(r, \theta, z)$, the basic Hagen-Poiseuille solution of the Navier-Stokes problem reads $\boldsymbol{u}_{b}=u_{r}^{b} \hat{\boldsymbol{r}}+u_{\theta}^{b} \hat{\boldsymbol{\theta}}+u_{z}^{b} \hat{z}=\left(1-r^{2}\right) \hat{\boldsymbol{z}}$. The Navier-Stokes equations for the velocity-pressure $\boldsymbol{u}=\left(u_{r}, u_{\theta}, u_{z}\right)-p$ perturbation fields are

$$
\begin{gathered}
\partial_{t} \boldsymbol{u}=-\nabla p+\frac{1}{R e} \Delta \boldsymbol{u}-(\boldsymbol{u} \cdot \nabla)\left(\boldsymbol{u}_{b}+\boldsymbol{u}\right)-\left(\boldsymbol{u}_{b} \cdot \nabla\right) \boldsymbol{u}+f \hat{\boldsymbol{z}}, \\
\nabla \cdot \boldsymbol{u}=0, \\
Q(\boldsymbol{u})=\int_{0}^{2 \pi} \int_{0}^{1}(\boldsymbol{u} \cdot \hat{z}) r \mathrm{~d} r \mathrm{~d} \theta=0, \\
\boldsymbol{u}(1, \theta, z ; t)=\mathbf{0}, \quad \boldsymbol{u}\left(r, \theta+2 \pi / n_{s}, z, t\right)=\boldsymbol{u}(r, \theta, z+2 \pi / \kappa, t)=\boldsymbol{u}(r, \theta, z, t),
\end{gathered}
$$

where an adjustable axial forcing $f=f(t)$ in (2.1) ensures the constant massflux constraint (2.3). The non-slip boundary condition at the wall and the axial and azimuthal periodicities are enforced by (2.4), where $\kappa$ is the non-dimensional wavenumber, here considered as an additional parameter selecting the axial periodicity of the flow structures. In this study, the axial wavenumber is varied in the range $\kappa \in[1.1,1.85]$, corresponding to pipe periodicities $\Lambda \in[3.40,5.71]$, in units of $D / 2$. This range covers all relevant solutions known so far up to $R e \simeq 1500$ while discarding subharmonic instabilities that might complicate the bifurcation scenario under examination at higher $R e$. The azimuthal wavenumber is set to $n_{s}=2$ throughout, meaning that only twofold azimuthally-periodic fields are considered. All solutions existing in this subspace are also solutions to the full Navier-Stokes equations with $n_{s}=1$, but the instabilities associated with perturbations without the $n_{s}=2$ symmetry are suppressed, rendering some of the solutions stable and thus accessible through time evolution.

For the spatial discretization of (2.1)-(2.4), we use a solenoidal spectral PetrovGalerkin scheme thoroughly described and tested by Meseguer \& Mellibovsky (2007). 
The method implicitly fulfils divergence-free and boundary conditions, and dispenses with the need for dealing with the pressure field. The velocity field is expanded following

$$
\begin{gathered}
\boldsymbol{u}(r, \theta, z ; t)=\sum_{l=-L}^{L} \sum_{n=-N}^{N} \exp \left(-\mathrm{i}\left(\kappa l z+n_{s} n \theta\right)\right) \boldsymbol{u}_{l n}(r ; t), \\
\boldsymbol{u}_{l n}(r ; t)=\sum_{m=0}^{M} a_{l n m}^{(1,2)}(t) \boldsymbol{v}_{l n m}^{(1,2)}(r),
\end{gathered}
$$

with $a_{l n m}^{(1,2)}$ being the complex expansion coefficients defining the vector state $\boldsymbol{a}$. In this way, the field decouples into its axial-azimuthal Fourier components $\boldsymbol{u}_{l n}$. As already noted, the expansion basis $\boldsymbol{v}_{l n m}(r)$, presented by Meseguer \& Mellibovsky (2007, (24)-(27)), is chosen so that boundary conditions (2.4) and solenoidality (2.2) are implicitly satisfied. For every $\operatorname{lnm}$, the three velocity components are related by the free-divergence condition, so that the expansion basis consists of two elements that we indicate by the superscript ' $(1,2)$ '. The spectral resolution, checked as adequate for the computations performed in this study, has been set to $L=16, N=12$ and $M=36$, corresponding to \pm 16 axial and \pm 24 azimuthal Fourier modes, and to 37 Chebyshev collocation points for the radial coordinate. Upon projection on the test basis $\tilde{\boldsymbol{v}}_{l n m}(r)$ (Meseguer \& Mellibovsky 2007, (32)-(35)), we are left with a system of ordinary differential equations for the complex-valued state vector $\boldsymbol{a}$ :

$$
\begin{gathered}
\boldsymbol{A} \dot{\boldsymbol{a}}=\left(\frac{1}{R e} \boldsymbol{B}_{1}+\boldsymbol{B}_{2}\right) \boldsymbol{a}-\boldsymbol{b}(\boldsymbol{a}, \boldsymbol{a})+f \boldsymbol{C}, \\
\boldsymbol{Q} \cdot \boldsymbol{a}=0,
\end{gathered}
$$

where the subscripts in Meseguer \& Mellibovsky (2007, (47)) have been omitted for simplicity and $\boldsymbol{B}$ has been decomposed into two separate contributions, $\boldsymbol{B}_{1}$ corresponding to the viscous dissipation term, with the dependence on $R e$ rendered explicit, and $\boldsymbol{B}_{2}$ to the linear advection terms. Here $\boldsymbol{C}$ is the projection of $\hat{z}$ onto the test basis and is therefore responsible for keeping the mass flux constant, represented by the linear scalar equation (2.8), by instantaneous adjustment of $f$.

The system (2.7) is evolved in time using a fourth-order linearly implicit method with $\Delta t=1 \times 10^{-2}$, where time is measured in units of $D /(4 U)$.

It will be useful to define some global properties of the flow that are independent of solid-body motions such as translation or rotation. One such quantity is the normalized energy of any given perturbation field $\boldsymbol{u}$, which is given by

$$
\varepsilon(\boldsymbol{u})=\frac{1}{2 \epsilon_{b}} \int_{0}^{2 \pi / \kappa} \mathrm{d} z \int_{0}^{2 \pi} \mathrm{d} \theta \int_{0}^{1} r \mathrm{~d} r \boldsymbol{u}^{*} \cdot \boldsymbol{u},
$$

with $\epsilon_{b}=\pi^{2} /(3 \kappa)$ being the energy of the basic flow and the asterisk symbolizing complex conjugation. This energy decouples exactly into the sum of its axial-azimuthal Fourier components:

$$
\varepsilon(\boldsymbol{u})=\sum_{l=-L}^{L} \sum_{n=-N}^{N} \varepsilon_{l n}=\varepsilon_{1 D}+\varepsilon_{2 \theta}+\varepsilon_{2 z}+\varepsilon_{3 D},
$$

where $\varepsilon_{l n}=\varepsilon\left(\boldsymbol{u}_{l n}\right)$ is the normalized energy associated with the $(l, n)$ axial-azimuthal Fourier mode. On the right of (2.10) the Fourier modes have been grouped in meaningful sets corresponding to the axisymmetric streamwise-independent component $\left(\varepsilon_{1 D}\right.$, 
mean flow component), the non-axisymmetric streamwise-independent component $\left(\varepsilon_{2 z}\right.$, energy signature of vortices and streaks), the axisymmetric streamwise-dependent component $\left(\varepsilon_{2 \theta}\right.$, mean wavy component) and the non-axisymmetric streamwisedependent component ( $\varepsilon_{3 D}$, purely three-dimensional energy).

Another global property is the mean axial pressure gradient that is required to drive the flow at constant mass flux, relative to its laminar flow value. It can be easily stated in terms of the adjusting intensity of the axial forcing $f$ and of $R e$ :

$$
(\nabla p)_{z}=1+\frac{\operatorname{Re} f}{4} .
$$

Finally, it will be useful to define the instantaneous axial and azimuthal phase speeds for any given velocity field. Their definition is clear for solid-body moving solutions, but can be extended to any velocity field by decomposing the time-evolving state vector into two contributions: one associated with solid-body motion and the other with a modulation. This decomposition can be made unique by minimizing, as a function of the phase speeds, at any given time, the two-norm of the modulational component:

$$
\min _{c_{z}, c_{\theta}}\left\|\boldsymbol{a}(t+\Delta t)-\boldsymbol{T}\left(c_{z} \Delta t\right) \boldsymbol{R}\left(c_{\theta} \Delta t\right) \boldsymbol{a}(t)\right\|,
$$

with $\boldsymbol{T}(\Delta z)$ and $\boldsymbol{R}(\Delta \theta)$ being the diagonal operators for translation and rotation, whose action on the state vector is multiplication of each component $a_{l n m}^{(1,2)}$ by $\mathrm{e}^{-\mathrm{i} \kappa l \Delta z}$ and $\mathrm{e}^{-\mathrm{i} n_{s} n \Delta \theta}$, respectively:

$$
\left.\begin{array}{rl}
(\boldsymbol{T}(\Delta z) \boldsymbol{a})_{l n m}^{(1,2)} & =a_{l n m}^{(1,2)} \mathrm{e}^{-\mathrm{i} \kappa l \Delta z}, \\
(\boldsymbol{R}(\Delta \theta) \boldsymbol{a})_{l n m}^{(1,2)} & =a_{l n m}^{(1,2)} \mathrm{e}^{-\mathrm{i} n_{s} n \Delta \theta} .
\end{array}\right\}
$$

For all rotating and travelling waves, $c_{z}$ and $c_{\theta}$ are constant, the minimum (2.12) is exactly zero, and they coincide with the axial and azimuthal phase speeds, respectively. The effective computation is done instantaneously by considering two consecutive time instants separated by $\Delta t$ and by applying a root-finding Newton method to the two-dimensional Jacobian of the norm in (2.12).

\subsection{Symmetries of the problem}

Pipe flow and its basic solution possess the $O(2) \times S O(2)$ symmetry, i.e. it is invariant under all azimuthal rotations about the axis and reflections with respect to all diametral planes, as well as under all axial translations. All travelling-wave families discovered so far are periodic and have axial and azimuthal dependence, thus breaking all continuous symmetries. While, in the azimuthal direction, the natural periodicity of the pipe renders all solutions invariant under rotations of $2 \pi$, travelling waves can have an additional discrete symmetry that renders them invariant under the cyclic group $C_{n_{s}}=\left\{R_{2 \pi}, R_{2 \pi / 2}, \ldots, R_{2 \pi / n_{s}}\right\}$ with $n_{s}$ being the azimuthal wavenumber and

$$
R_{\alpha}(\boldsymbol{u})(\boldsymbol{x})=R_{\alpha}(u, v, w)(r, \theta, z ; t)=(u, v, w)(r, \theta+\alpha, z ; t) .
$$

In the axial direction, on top of the trivial shift by a wavelength, the axial symmetry rupture gives rise to a special type of time periodicity, in the sense that the time dependence is a bare drift in the direction of the $S O(2)$ symmetry. Travelling waves are therefore best described as relative equilibria that possess the continuous spacetime symmetry

$$
(u, v, w)_{t w}(r, \theta, z ; t)=(u, v, w)_{t w}\left(r, \theta, z-c_{z} t ; 0\right),
$$


where $c_{z}$ is the axial drift speed. In a comoving reference frame travelling downstream with speed $c_{z}$, travelling waves appear as stationary solutions. Near a relative equilibrium, the drift dynamics is trivial and decouples from the dynamics orthogonal to the drift. As a result, bifurcations of relative equilibria can be analysed in two steps, first describing the bifurcations associated with the orthogonal dynamics, then adding the corresponding drift along the travelling direction (Krupa 1990).

There are a couple of additional non-trivial symmetries that leave some of the known travelling waves unaltered. The first is a mirror symmetry with respect to $n_{s}$ diametral planes:

$$
M \boldsymbol{u}(\boldsymbol{x})=M(u, v, w)\left(r, \theta_{i}+\theta, z ; t\right)=(u,-v, w)\left(r, \theta_{i}-\theta, z ; t\right),
$$

with $\theta_{i}=\theta_{0}+i \pi / n_{s}, i=\left\{0,1, \ldots, n_{s}-1\right\}$, and $\theta_{0}$ parametrizing the azimuthal degeneracy of solutions.

The second symmetry, characteristic of the travelling-wave family studied here, is a combined shift-reflect symmetry. Solutions invariant under this symmetry operation,

$$
S \boldsymbol{u}(\boldsymbol{x})=S(u, v, w)\left(r, \theta_{i}+\theta, z ; t\right)=(u,-v, w)\left(r, \theta_{i}-\theta, z+\pi / \kappa ; t\right),
$$

are left unaltered when shifted half a wavelength downstream and then reflected with respect to any of $n_{s}$ diametral planes tilted with $\theta_{i}$.

A number of highly symmetric solutions possessing both symmetries (Pringle et al. 2009) have been identified and continued to very low $R e$. Some of the less symmetric waves are known to emerge from symmetry-breaking bifurcations of these highly symmetric waves.

All symmetries can be enforced in the computations. This applies to time evolution, computation of relative equilibria using Newton iteration and stability analysis of relative equilibria via Arnoldi iteration. Nevertheless, we have chosen to implement, in addition to the translational invariance due to axial periodicity, only a restriction on the azimuthal wavenumber $n_{s}$ to avoid inevitable instability to azimuthally non-periodic flows. Therefore, all other symmetries could be broken dynamically during time evolution, but we did not find any evidence for that. All computations spontaneously restored and preserved the shift and reflect symmetry.

\subsection{Computation of relative equilibria, continuation and stability analysis}

The spectral method discussed in $\S 2.1$ is extremely versatile and very well suited for straightforward coding of a Newton method for the computation of relative equilibria, for their continuation in parameter space and for linear-stability analysis using Arnoldi iteration. Some of these usages of the spectral method were briefly described by Meseguer et al. (2007) but will be developed further here.

\subsubsection{Computation of travelling/rotating waves}

The spectral representation of a generic travelling and/or rotating wave reduces to

$$
\boldsymbol{a}(t)=\boldsymbol{T}\left(c_{z} t\right) \boldsymbol{R}\left(c_{\theta} t\right) \boldsymbol{a}_{t w}
$$

where $\boldsymbol{a}_{t w}$ is the state vector corresponding to the time-independent structure of the wave. The scalar quantities $c_{z}$ and $c_{\theta}$ are the axial and azimuthal advection speeds. The travelling-waves family studied here has no rotation and, therefore, $c_{\theta}=0$. 
Substitution of (2.18) into (2.7) and (2.8) yields a nonlinear system of algebraic equations

$$
\left.\begin{array}{l}
\boldsymbol{F}\left(\boldsymbol{a}_{t w}, c_{z}, c_{\theta}, f\right)=\mathbf{0}, \\
\boldsymbol{Q} \cdot \boldsymbol{a}_{t w}=0, \\
\phi_{z}\left(\boldsymbol{a}_{t w}\right)=\phi_{z}\left(\boldsymbol{a}^{0}\right), \\
\phi_{\theta}\left(\boldsymbol{a}_{t w}\right)=\phi_{\theta}\left(\boldsymbol{a}^{0}\right),
\end{array}\right\}
$$

where the last two scalar equations impose the phase on any two suitable coefficients so as to lift the translational and rotational degeneracy of the solution. The vector function $\boldsymbol{F}$ is given by

$$
\boldsymbol{F}\left(\boldsymbol{a}_{t w}, c_{z}, c_{\theta}, f\right)=\left(\frac{1}{R e} \boldsymbol{B}_{1}+\boldsymbol{B}_{2}-\boldsymbol{A}\left(c_{z} \hat{T}+c_{\theta} \hat{R}\right)\right) \boldsymbol{a}_{t w}-\boldsymbol{b}\left(\boldsymbol{a}_{t w}, \boldsymbol{a}_{t w}\right)+f \boldsymbol{C},
$$

with $\hat{T}$ and $\hat{R}$ being the diagonal operators for infinitesimal translation and rotation, whose effect on a state vector is multiplication of each element by $-\mathrm{i} \kappa l$ and $-\mathrm{i} n_{s} n$, respectively:

$$
\left.\begin{array}{l}
(\hat{T} \boldsymbol{a})_{l n m}^{(1,2)}=-\mathrm{i} \kappa l a_{l n m}^{(1,2)} \\
(\hat{R} \boldsymbol{a})_{l n m}^{(1,2)}=-\mathrm{i} n_{s} n a_{l n m}^{(1,2)}
\end{array}\right\}
$$

These operators arise from the time derivative in (2.7) when applied to (2.18) and correspond to the linearization of $\boldsymbol{T}$ and $\boldsymbol{R}$ of (2.13) with respect to the scalar variable.

The system (2.19) can be solved via Newton iteration. At step $k$, the linear system to be solved takes the form

$$
\left[\begin{array}{cccc}
D_{a} \boldsymbol{G}^{k} & D_{c_{z}} \boldsymbol{G}^{k} & D_{c_{\theta}} \boldsymbol{G}^{k} & \operatorname{Re} \boldsymbol{B}_{1}^{-1} \boldsymbol{C} \\
\boldsymbol{\phi}_{z}^{T} & 0 & 0 & 0 \\
\boldsymbol{\phi}_{\theta}^{T} & 0 & 0 & 0 \\
\boldsymbol{Q}^{T} & 0 & 0 & 0
\end{array}\right]\left(\begin{array}{c}
\Delta \boldsymbol{a}^{k} \\
\Delta c_{z}^{k} \\
\Delta c_{\theta}^{k} \\
\Delta f^{k}
\end{array}\right)=-\left(\begin{array}{c}
\boldsymbol{G}^{k} \\
0 \\
0 \\
0
\end{array}\right),
$$

where Stokes preconditioning (multiplication by $\operatorname{Re} \boldsymbol{B}_{1}^{-1}$ from the left) has been applied to the first row to assist convergence (Mamun \& Tuckerman 1995). Accordingly, $\boldsymbol{G}^{k}=\operatorname{Re} \boldsymbol{B}_{1}^{-1} \boldsymbol{F}\left(\boldsymbol{a}^{k}, c_{z}^{k}, c_{\theta}^{k}, f^{k}\right)$. The terms appearing in the first row on the left-hand side correspond to the Jacobian of $\boldsymbol{G}$, denoted $D_{x} \boldsymbol{G}$, evaluated at step $k$ :

$$
\begin{aligned}
& D_{\boldsymbol{a}} \boldsymbol{G}^{k}=\boldsymbol{I}+\operatorname{Re} \boldsymbol{B}_{1}^{-1} \boldsymbol{B}_{2}-\operatorname{Re} \boldsymbol{B}_{1}^{-1} \boldsymbol{A}\left(c_{z}^{k} \hat{T}+c_{\theta}^{k} \hat{R}\right)-\operatorname{Re} \boldsymbol{B}_{1}^{-1} D_{\boldsymbol{a}} \boldsymbol{b}\left(\boldsymbol{a}^{k}\right), \\
& D_{c_{z}} \boldsymbol{G}^{k}=-\operatorname{Re} \boldsymbol{B}_{1}^{-1} \boldsymbol{A} \hat{T} \boldsymbol{a}^{k} \\
& D_{c_{\theta}} \boldsymbol{G}^{k}=-\operatorname{Re} \boldsymbol{B}_{1}^{-1} \boldsymbol{A} \hat{R} \boldsymbol{a}^{k}
\end{aligned}
$$

where $D_{\boldsymbol{a}} \boldsymbol{b}\left(a^{k}\right) \Delta \boldsymbol{a}^{k}$, the linearized advection term, is computed in physical space as $\left(\Delta \boldsymbol{u}^{k} \cdot \nabla\right) \boldsymbol{u}_{t w}{ }^{k}+\left(\boldsymbol{u}_{t w}{ }^{k} \cdot \nabla\right) \Delta \boldsymbol{u}^{k}$ and then projected back to spectral space (Meseguer \& Mellibovsky 2007). Arrays $\boldsymbol{\phi}_{z}$ and $\boldsymbol{\phi}_{\theta}$ correspond to the linearization of the phase prescription of any two coefficients, as long as they are associated with basis elements that entrain axial and azimuthal structures, respectively. In the notation of Meseguer \& Mellibovsky (2007), we have chosen to fix the phase of $a_{100}^{(2)}$ and $a_{010}^{(2)}$ to lift the axial and azimuthal degeneracies of the solutions, respectively. The incremental vector which the Newton method requires solving for at each step is defined as $\Delta x^{k}=x^{k+1}-x^{k}$ and provides the new guess for step $k+1$. 
The solution of the linear system at every Newton step needs only to be approximated by using inexact Krylov iteration. Generalized minimal residuals (see Frayssé et al. 2003, for the implementation of GMRES we use) and stabilized biconjugate gradient methods (BiCGSTab) can be easily implemented (see e.g. Quarteroni, Sacco \& Saleri 2007, and references therein). Convergence to the exact solution of the nonlinear equations to numerical accuracy is guaranteed provided that a sufficiently good initial guess $\left(\boldsymbol{a}^{0}, c_{z}^{0}, c_{\theta}^{0}, f^{0}\right)$ is fed into the iteration. Convergence can be enhanced by appropriate damping (Quarteroni et al. 2007), used in this study, or trust-region techniques (Dennis \& Schnabel 1996).

\subsubsection{Continuation of solutions}

It is fairly easy to extend the Newton method just described to the continuation in $R e$ of any branch of solutions. The explicit dependence of the system (2.19) on $R e$ allows this parameter to be treated as an additional unknown. Orthogonality to the Jacobian at some prescribed distance (pseudo-arclength) from the departing solution provides the additional equation needed (Sanchez, Marques \& Lopez 2002). This continuation technique is capable of following branches around turning points, which makes it very convenient in the presence of saddle-node bifurcations. Continuation in the other parameter $\kappa$ is not as straightforward. The complex dependence of the linear contribution on $\boldsymbol{F}$ of $\kappa$ requires recomputing the linear operators at every single Krylov iteration, which renders the method very costly. We have chosen to continue it manually by simply using the Newton method with discrete jumps in $\kappa$, small enough so that the converged solution at the previous $\kappa$ constitutes a sufficiently good guess. This procedure cannot go around turning points, but combined with pseudo-arclength continuation in $R e$, the full family of travelling waves can be computed.

\subsubsection{Stability analysis}

Special care must be taken when analysing the linear stability of rotating/travelling waves. Such waves have two equivalent representations in the constant pressure gradient and in the constant flow-rate frames. This is so because their solidbody motion modifies neither the mass flux nor the mean axial pressure gradient. However, their destabilization does not necessarily preserve these two global quantities simultaneously, meaning that linear stability will explicitly depend on which of the two is prescribed as constant. Since we have chosen to study pipe flow at a constant flow rate, we must accordingly allow for pressure variation. Let us consider an infinitesimal perturbation $\left(\boldsymbol{v}_{\boldsymbol{a}}, v_{f}\right)$ on a given travelling/rotating wave $\left(\boldsymbol{a}_{t w}, f_{t w}\right)$ in a reference frame moving with the wave:

$$
\left.\begin{array}{l}
\boldsymbol{a}=\boldsymbol{T}\left(c_{z} t\right) \boldsymbol{R}\left(c_{\theta} t\right)\left(\boldsymbol{a}_{t w}+\boldsymbol{v}_{\boldsymbol{a}} \mathrm{e}^{\lambda t}\right) \\
f=f_{t w}+v_{f} \mathrm{e}^{\lambda t}
\end{array}\right\}
$$

The factor $\mathrm{e}^{\lambda t}$ is intended to capture the linear evolution of the perturbation in the vicinity of the relative equilibrium. In this sense, $\left(\boldsymbol{v}_{\boldsymbol{a}}, v_{f}\right)$ is an eigenvector of the eigenvalue $\lambda$, which in general can be complex. Introducing (2.24) into (2.7) and (2.8) yields the evolution equation for the perturbation, which can be linearized around the wave solution by discarding all second-order terms:

$$
\left.\begin{array}{rl}
\left(\frac{1}{R e} \boldsymbol{B}_{1}+\boldsymbol{B}_{2}-\boldsymbol{A}\left(c_{z} \hat{T}+c_{\theta} \hat{R}\right)-D_{a} \boldsymbol{b}\left(\boldsymbol{a}_{t w}\right)\right) \boldsymbol{v}_{\boldsymbol{a}}+\boldsymbol{C} v_{f} & =\lambda \boldsymbol{A} \boldsymbol{v}_{\boldsymbol{a}}, \\
\boldsymbol{Q} \cdot \boldsymbol{v}_{\boldsymbol{a}} & =0 .
\end{array}\right\}
$$


The system (2.25) constitutes a constrained generalized eigenproblem which can be expressed in matrix form as

$$
\mathscr{A} x=\lambda \mathscr{M} x
$$

where

$$
\left.\begin{array}{c}
\mathscr{A}=\left[\begin{array}{cc}
\frac{1}{R e} \boldsymbol{B}_{1}+\boldsymbol{B}_{2}-\boldsymbol{A}\left(c_{z} \hat{T}+c_{\theta} \hat{R}\right)-D_{a} \boldsymbol{b}\left(\boldsymbol{a}_{t w}\right) & \boldsymbol{C} \\
\boldsymbol{Q}^{\mathrm{T}} & 0
\end{array}\right], \\
\mathscr{M}=\left[\begin{array}{cc}
\boldsymbol{A} & \mathbf{0} \\
\mathbf{0}^{\mathrm{T}} & 0
\end{array}\right] \quad \text { and } \quad \boldsymbol{x}=\left(\begin{array}{c}
\boldsymbol{v}_{a} \\
\boldsymbol{v}_{f}
\end{array}\right) .
\end{array}\right\}
$$

Since we are only interested in leading eigenvalues (those with larger real part) while usual iterative methods are good at capturing dominant eigenvalues (those with larger modulus), we solve (2.26) in the shift-invert mode (Meerbergen, Spence \& Roose 1994):

$$
(\mathscr{A}-\sigma \mathscr{M})^{-1} \mathscr{M} \boldsymbol{x}=v \boldsymbol{x}, \quad v=\frac{1}{\lambda-\sigma},
$$

with a real shift $\sigma$ applied to avoid the neutrally stable double eigenvalue at the origin corresponding to infinitesimal rotations and translations.

The method used is Arnoldi iteration with Krylov-based iteration for the solution of the linear system involved at each step (Freitag 2007). We use the robust routines by Lehoucq \& Scott (1996). Stokes preconditioning is employed as for the Newton method to speed up convergence.

\subsection{Normal form reduction}

We will be concerned here with the stability and bifurcation of travelling-wave-type solutions. Given that they appear as periodic solutions in a stationary frame, a Poincare section should be defined and the bifurcation analysis carried out for the map defined by the resulting Poincare application. In this setting, travelling waves would be seen as fixed points and modulated waves as periodic orbits. It is common practice, though, to approximate the map to any desired accuracy with a system of differential equations (see Guckenheimer \& Holmes 1983, chapter 4). Bifurcations of maps can thus be studied by constructing continuous-time normal forms. This comes out naturally when studying the stability of relative equilibria in a comoving reference frame (2.24). In the close neighbourhood of the relative (or degenerate) equilibrium, such an analysis is perfectly accurate (Rand 1982; Krupa 1990). These travelling waves may typically undergo Hopf bifurcations. The bifurcation is considered a Hopf bifurcation from a relative equilibrium rather than a Neimark-Sacker (Hopf bifurcation for maps) because of the special type of time-dependence involved. The bifurcating quasi-periodic solution is a modulated travelling wave which is just a periodic orbit in the comoving frame in which the travelling wave is stationary. Modulated waves that bifurcate via Hopf bifurcations from drifting waves in systems with $S O(2)$ symmetry were classified in terms of the spatial symmetry of the drifting wave by Rand (1982). The classification was completed by Golubitsky, LeBlanc \& Melbourne (2000) to include the spatiotemporal symmetry of the modulated wave. The appropriate way to investigate these solutions is to study the dynamics normal to the group orbit (Krupa 1990), which in our case is equivalent to considering the dynamics in a moving reference frame in which the travelling wave is stationary.

A problem arises when, for given values of the parameters, coexistence of several waves occurs and, possibly, of one or more modulated waves. In this case, each 
wave will generally drift with its own phase speed, so that there is no unique moving reference frame in which their degeneracy could be lifted simultaneously. This is easily overcome by restating the problem with the use of Poincare sections, combined with continuous-time interpolation of the corresponding maps. This of course only solves the problem as long as the limits of validity of the interpolation are not infringed. In particular, special care should be taken when studying the dynamics in the vicinity of homoclinic orbits, where arbitrarily small perturbations can lead to qualitatively different behaviour due to manifolds entanglement. In the absence of translational symmetry, complex dynamics would arise, i.e. horseshoes (Smale 1967; Guckenheimer \& Holmes 1983). However, it is possible to show that, due to the translational symmetry of the pipe, if a point lies on both the stable and the unstable manifolds of a relative (drifting) saddle such as a lower-branch travelling wave, then the unstable manifold is contained within the stable manifold (no transversal intersection is allowed) and complex dynamics does not occur (Chossat \& Lauterbach 2000).

It will be argued that, in the problem at hand, there are only two active modes (bifurcating eigenvalues) in the range of parameters studied, all other modes being slaved to these two via the centre manifold theorem. This, added to the translational symmetry of the pipe, results in the ability of capturing the dynamics within this region of parameter space with a pair of amplitude equations:

$$
\left.\begin{array}{l}
\dot{x}=F_{1}(x, y), \\
\dot{y}=F_{2}(x, y),
\end{array}\right\}
$$

where $x$ and $y$ are real amplitudes related to velocity fields in (2.1). The simplest expansion for $F_{1}$ and $F_{2}$ that is compatible with the dynamics observed upon integration of (2.7) is the subject of normal form theory and will be developed along the analysis.

\section{Results}

\subsection{Travelling waves}

The special stability properties of the specific family of travelling waves in the twofold periodic subspace single them out for the kind of analysis performed in this study. These waves, whose existence relies on the self-sustaining process (SSP; Waleffe 1995, 1997), were first computed by Faisst \& Eckhardt (2003) and Wedin \& Kerswell (2004) using volume forcing homotopy. They are characterized by discrete twofold azimuthal periodicity $\left(C_{2}\right.$, i.e. $\left.n_{s}=2\right)$ and a combined shift-reflect symmetry $(S)$.

For a given $\kappa$ and above a certain critical $R e$, these waves appear in pairs, one that is closer to the laminar solution, the other further away in all respects and whatever the property examined (e.g. friction factor, energy contents and distance in phase space). They are accordingly tagged as a lower-branch and an upperbranch solution, respectively. Figure 1 (see supplementary movies available at journals.cambridge.org/flm) shows axial velocity contours together with in-plane velocity vectors on four cross-sections spanning half a wavelength for $\kappa=1.52, R e=1600$ upper-branch (figure 1, top) and lower-branch (figure 1, bottom) travelling waves. The discrete twofold azimuthal periodicity can be seen in all cross-sections. Also, the combined shift-reflect symmetry can be grasped by comparing the $z=0$ and $z=\Lambda / 2$ cross-sections of either travelling wave. The reflection plane is tilted by $\theta_{0} \simeq \pi / 4$. The complementary half-wavelength is not shown, as it can be constructed by mere 

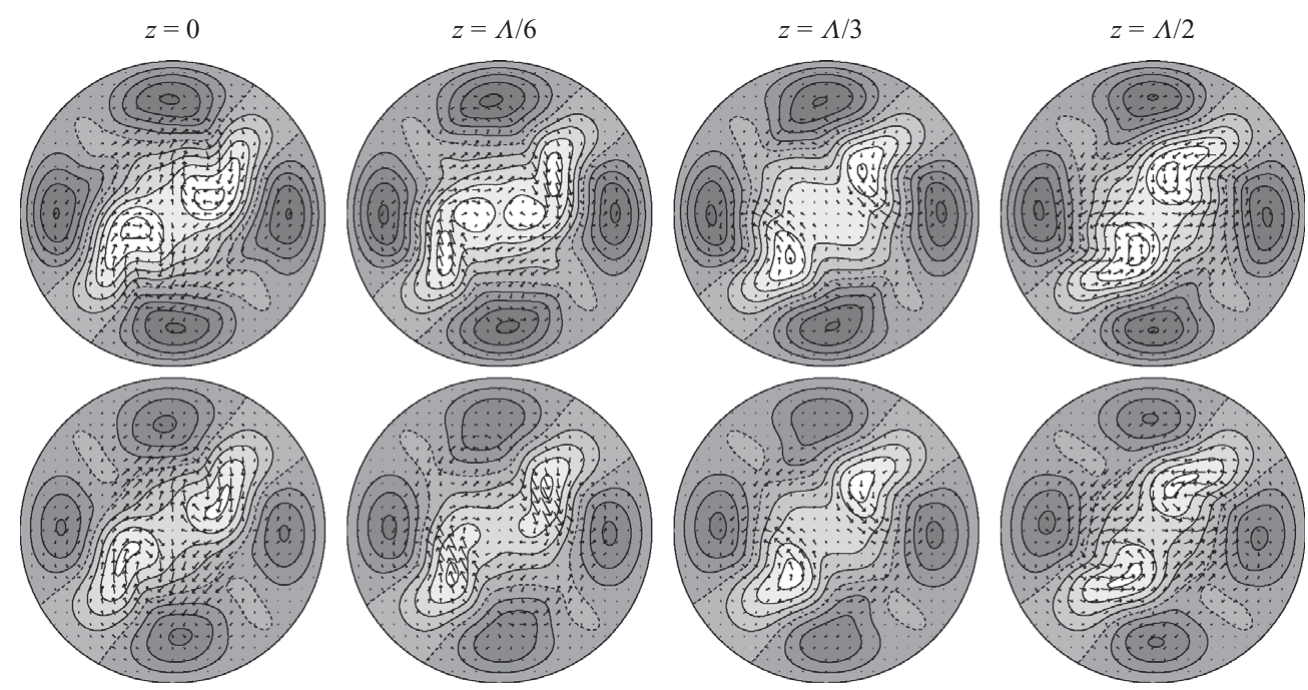

FIGURE 1. Cross-sections spanning half a wavelength $(\Lambda / 2=\pi / \kappa)$ of the upper-branch (top) and the lower-branch (bottom) travelling waves for $\kappa=1.52, R e=1600$. Axial perturbation velocity contour lines are equispaced with intervals of $\Delta u_{z}=0.1 U$. Regions where the axial velocity is faster or slower than the laminar parabolic profile are coded in dark and light, respectively, with the zero contour line marked by a dashed line. In-plane velocity vectors are also shown.

(a)

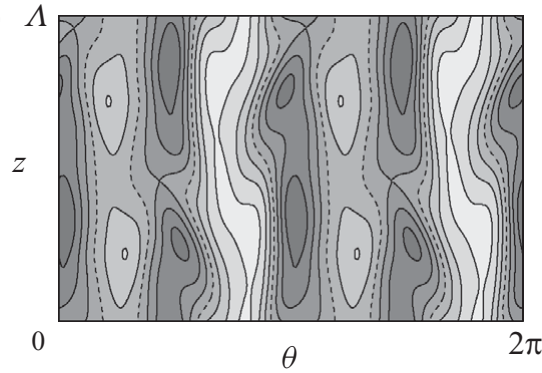

(b)

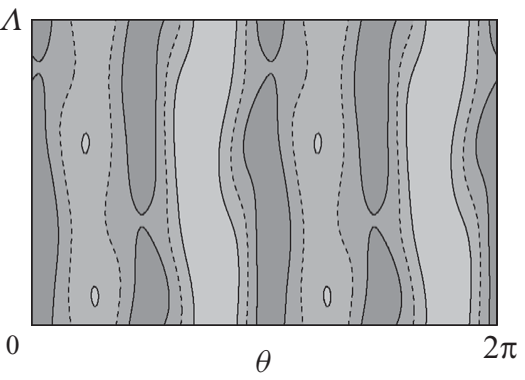

FIGURE 2. Radial sections at $r=0.65$ of $(a)$ the upper-branch and $(b)$ the lower-branch travelling waves for $\kappa=1.52, R e=1600$. Radial velocity contour lines are equispaced with intervals of $\Delta u_{r}=0.0072 U$. Regions where the flow moves towards the walls or the pipe centre are shaded dark and light, respectively.

reflection of the given cross-sections. The travelling waves shown are representative of the full family in that they are characterized by wobbling low-speed streaks (light shading) in the pipe's central region and fairly streamwise-independent high-speed streaks in the wall region (dark). Leaving aside weak secondary vortices, a couple of strong vortex pairs can be identified from the velocity vectors. In each of the pairs, the vortices are not of equal intensity, but alternate in strength along the axial direction. Except for the higher axial velocities and the slightly wider low-speed streaks, not much distinguishes the upper-branch solution from the lower-branch solution.

Radial-velocity contour lines on a radial plane at $r=0.65$ have been plotted in figure 2 for both travelling waves. The difference between upper-branch and lowerbranch waves becomes clearer, the former involving much larger radial transport. These sections display clearly both the azimuthal periodicity and the shift-reflect symmetry of the waves. Structures resembling these and other travelling waves have 

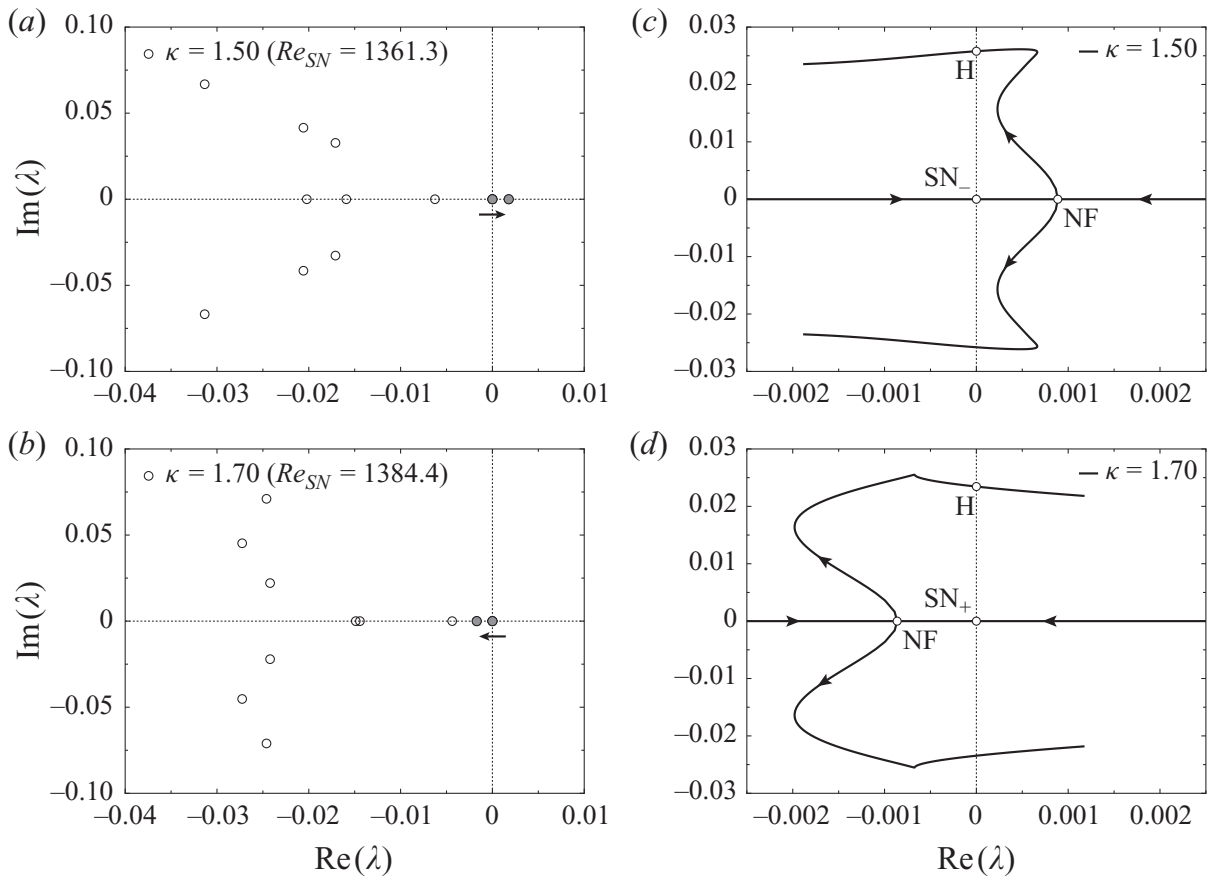

FiguRE 3. Rightmost eigenvalues of $(a) \kappa=1.50$ and $(b) \kappa=1.70$ travelling waves at their respective saddle-node bifurcation (SN). The direction of the zero-crossing from lower to upper branch is indicated by an arrow. The eigenvalues marked by filled circles have been followed across the saddle-node point and beyond for both $(c) \kappa=1.50$ and $(d) \kappa=1.70$. Saddle-node $(\mathrm{SN})$, node-focus (NF) and Hopf (H) points are identified by circles.

been observed experimentally in developed turbulence (Hof et al. 2004) as well as in numerical simulation (Kerswell \& Tutty 2007; Schneider et al. 2007a; Willis \& Kerswell 2008).

These travelling waves appear in saddle-node bifurcations starting from $R e \simeq 1358.5$ for the optimal wavenumber $\kappa \simeq 1.55$ (Wedin $\&$ Kerswell 2004). Lower-branch solutions exhibit a single unstable eigenmode when considered in the azimuthal subspace where they exist, which makes them accessible through time integration if appropriately combined with edge-tracking techniques. In this sense, they are edge states (Schneider et al. 2007b) of the twofold azimuthally-periodic pipe, and they constitute an example of non-uniqueness of the edge state, since they coexist as attractors within the critical threshold, and for some range of $\kappa$ and $R e$, with another family of travelling waves that are both shift-reflect and mirror symmetric and that have also a single unstable direction (Duguet et al. 2008b). The stable manifold of these waves separates, albeit only locally, initial conditions that decay uneventfully from others that undergo turbulent transients. For this reason, lower-branch solutions are believed to play a central role in the transition process.

As already pointed out, lower-branch solutions, when continued down in $R e$, merge with upper-branch solutions in a saddle-node-type bifurcation. Around the saddle-node point, a real eigenvalue must cross the imaginary axis, either adding or subtracting an unstable direction. As a matter of fact, both scenarios occur and the stability properties of upper-branch solutions depend on the axial wavenumber $\kappa$. Upper-branch long waves are unstable, whilst short waves are stable. The spectra of a long $(\kappa=1.50)$ and a short $(\kappa=1.70)$ wave are shown in figures $3(a)$ and $3(b)$, 

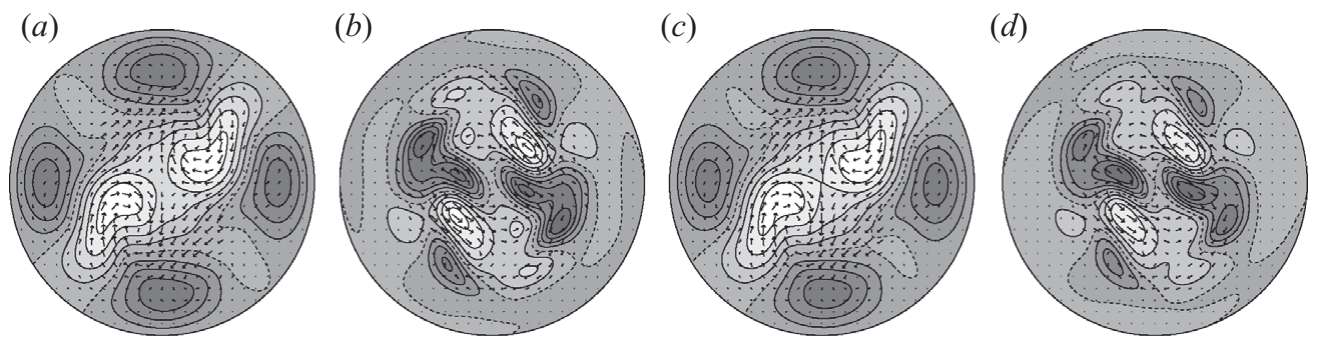

FIGURE 4. (a) $\kappa=1.50$ and $(c) \kappa=1.70$ travelling waves at their respective saddle-node bifurcation points (SN in figure 3). Also shown are the bifurcating eigenfunctions for (b) $\kappa=1.50$ and $(d) \kappa=1.70$. Axial perturbation velocity contour lines are equispaced in intervals of $\Delta u_{z}=0.1 U$.

respectively, at their respective saddle-node points. The arrow below the bifurcating eigenvalue points in the crossing direction when followed from lower to upper branch. For $\kappa=1.50$, the solution switches from one to two unstable directions, while for $\kappa=1.70$ it switches from one to nil. Figures $3(c)$ and $3(d)$ show the trajectories followed by the two rightmost eigenvalues (filled circles in figure $3 a, b$ ) when continued in $R e$ across the saddle-node and beyond, for $\kappa=1.50$ and $\kappa=1.70$, respectively. In both cases, the eigenvalues collide and become complex in a node-focus transition, but they can do so at either side of the imaginary axis. Such a transition preserves the stability properties of the solution, but trajectories around it start spiralling. When followed further to higher Re along the upper branch, long and short solutions undergo a stabilizing or destabilizing Hopf bifurcation, respectively, implying the existence of modulated waves (relative periodic orbits).

Cross-sections of both travelling waves at their respective saddle-node points have been plotted in figures $4(a)$ and $4(c)$, together with their corresponding crossing eigenfunctions (figures $4 b$ and $4 d$ ). It is clear from the contour plots that the effect of varying $\kappa$ on the solutions does not affect the flow structure dramatically, aside from the stretching and the stability properties. It is also remarkable how the eigenfunctions corresponding to two different eigenvalues (figure $4 b, d$ ) appear so similar to each other, even for different $\kappa$. This is a signature of the tendency to become parallel (linearly dependent on one another), which ultimately leads to collision in a nodefocus transition. Both eigenfunctions preserve the same symmetries as the travelling waves, so that all solutions arising from their bifurcation must necessarily possess the same symmetries.

Wavenumber continuity suggests that there might be an intermediate value of $\kappa$ for which the node-focus point can be brought to the origin of the complex plane. Appropriate tuning of $\kappa$ and $R e$ would therefore help unfold a codimension-two double-zero bifurcation, a complete study of which is due to Takens (1974) and Bogdanov (1975). The zero eigenvalue has algebraic multiplicity 2 and geometric multiplicity 1 , leading to a $2 \times 2$ Jordan block that is at the heart of the analysis. As a matter of fact, the trajectories of the complex eigenvalues, following a left opening parabola and an eventual right turn, indicate that for a certain range of $\kappa$ an additional pair of crossings will take place, thus implying additional Hopf bifurcations closely related to the appearance of periodic orbits in a double-zero bifurcation.

\subsection{Modulated travelling waves}

Figure 5(a) depicts the trajectories of the relevant eigenvalues for $\kappa=1.52$. They are extremely close to those observed for $\kappa=1.50$, except that the nose is becoming 

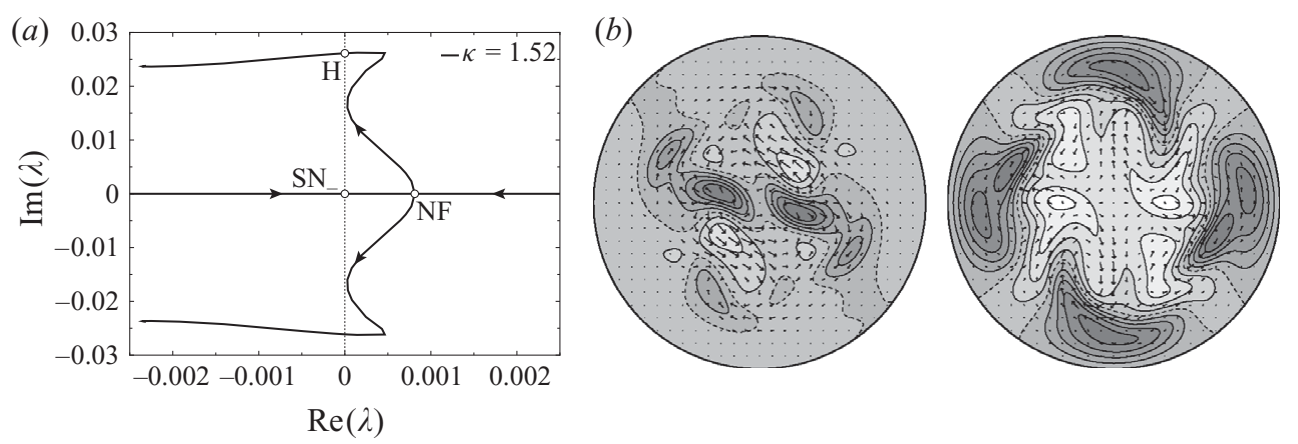

FIGURE 5. (a) Trajectories of the two rightmost eigenvalues for $\kappa=1.52$. (b) Real (left) and imaginary (right) parts of the corresponding eigenvectors at the Hopf bifurcation $(\mathrm{H}$, $R e=1775.4)$.

tangent to the imaginary axis. The implications of this will be discussed later. Let us focus on the Hopf bifurcation for the time being. The crossing takes place at $R e=1775.4$ on the upper branch, with the arrows pointing towards higher $R e$. The real and imaginary parts of the bifurcating eigenfunction are shown in figure $5(b)$ only for completeness, since it is difficult to extract from them relevant information on the nature of the instability that they carry. As already noted, the instability does not break any of the symmetries exhibited by the bifurcating travelling wave.

The existence of a Hopf bifurcation implies the appearance of a time-periodic solution, in this case a modulated wave or relative periodic orbit. Since the crossing is leftwards as $R e$ is increased, it necessarily carries with it a stabilization of the upper-branch travelling wave, which was unstable at onset. The Hopf bifurcation happens to be supercritical in the sense that the first Lyapunov coefficient is negative (Kuznetsov 1995). This we infer from the fact that time evolution unveils a stable branch of modulated waves pointing towards decreasing $R e$.

Simple time evolution starting from the $(\kappa, R e)=(1.52,1600)$ unstable upper-branch travelling wave (figure 1, top) departs in an oscillatory fashion, as predicted by the complex pair of unstable eigenvalues, to nonlinearly converge onto a modulated wave.

Travelling waves, which are degenerate or relative equilibria, and solutions bifurcating from them have a pure frequency associated with the advection speed (solid-body translation). Local quantities such as point velocities or pressures necessarily reflect on this frequency. However, since it reflects a neutral direction, it can be suppressed by restating the problem in a comoving frame. Global quantities such as modal energies or volume-averaged fields naturally overlook solid-body rotation and translation, making them suitable for a decoupled analysis in the direction orthogonal to the degenerate drift. Thus, as justified before, the bifurcation analysis of travelling waves can be carried out analogously to that of fixed points, as long as special care is taken in the neighbourhood of homoclinic connections (Rand 1982; Golubitsky et al. 2000). The Hopf bifurcation adds a modulational frequency to the pure translational frequency. As a result, global quantities cease to be constant and oscillate with this frequency. The Fourier transform of the energy contained in non-axisymmetric streamwise-dependent modes $\left(\varepsilon_{3 D}\right)$ has been plotted in figure $6(a)$, with the time signal shown in the inset. The spectrum reveals that the solution has a strong mean component and a peak angular frequency at $\omega_{0}=0.0219$, corresponding to a period $T=2 \pi / \omega_{0}=286.7$. This period is extremely long when compared with the 

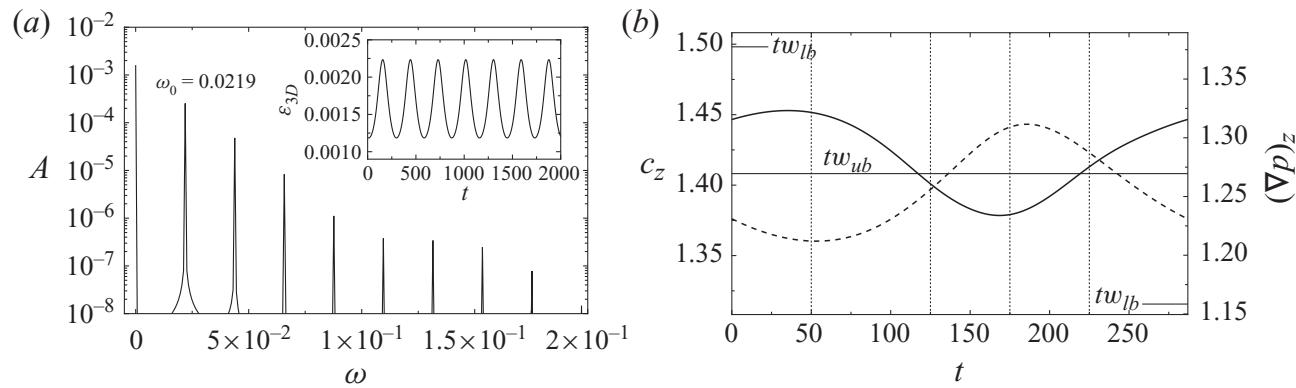

FiguRE 6. (a) Fourier transform of the non-axisymmetric streamwise-dependent modal energy contents $\left(\varepsilon_{3 D}\right)$ time stamp for the $(\kappa, R e)=(1.52,1600)$ modulated wave. The time signal is plotted in the inset. (b) Advection speed $\left(c_{z}\right.$, solid line) and mean axial pressure gradient normalized by its laminar value $\left((\nabla p)_{z}\right.$, dashed line) along a full period. Values for the $(\kappa, R e)=(1.52,1600)$ upper-branch $\left(t w_{u b}\right)$ and lower-branch $\left(t w_{l b}\right)$ travelling waves are indicated by horizontal lines.

streamwise advection time scale, which is of the order of $2 \pi /\left(\kappa c_{z}\right) \sim 3$. The signal is not strictly sinusoidal and some energy is spread among a number of harmonics of $w_{0}$. A full period of the axial advection speed $\left(c_{z}\right)$ and the mean axial pressure gradient normalized by its laminar value $\left((\nabla p)_{z}\right)$ has been plotted in figure $6(b)$. Both are global quantities and, as expected, oscillate with the modulational frequency $\omega_{0}$. The horizontal line indicates the constant value of these quantities for the unstable upperbranch travelling wave from which the modulated wave bifurcates. The modulated wave orbits in phase space around the travelling wave, so that the instantaneous values of the former oscillate around those of the latter. The differences between time-averaged quantities and travelling wave values are purely due to nonlinear effects, since for this $R e$ and $\kappa$ we are already some distance away from the linear influence of the instability.

Figure 7 (see supplementary movie) depicts a couple of cross-sections $(z=0$, figure 7 , top; $z=\Lambda / 4$, figure 7 , bottom) of the modulated wave at four different time instants along one period (from left to right, $t=50,125,175$ and 225). The modulation is obvious from the series of snapshots yet not very prominent, as could be expected from the mild oscillation (within $\pm 5 \%$ ) of the time series in figure $6(b)$. Comparison with figure 1 (top) reveals that the overall flow structure of the travelling wave is preserved on average but is now dependent on time.

Radial sections showing radial velocity contours have been plotted in figure 8 (see supplementary movie) to convey in a clear way the modulation of the wave along a full period. It also confirms that all symmetries are preserved. It will soon become clear that the snapshot at $t=50$ is about the closest approach to the lowerbranch travelling wave, while all of them are approximately equally distant from the upper-branch wave.

Starting time evolution from the lower-branch travelling wave (figure 1, bottom) fails to reach the modulated wave. Trajectories issued from the former either relaminarize uneventfully or make a long detour around both the upper-branch travelling wave and the modulated wave to end up relaminarizing. This suggests that some object might be shielding the stable modulated wave, thus preventing convergence onto it.

Using time evolution and edge-tracking refinement (Skufca et al. 2006; Schneider et al. 2007b), where the laminar and turbulent asymptotic states have been replaced 

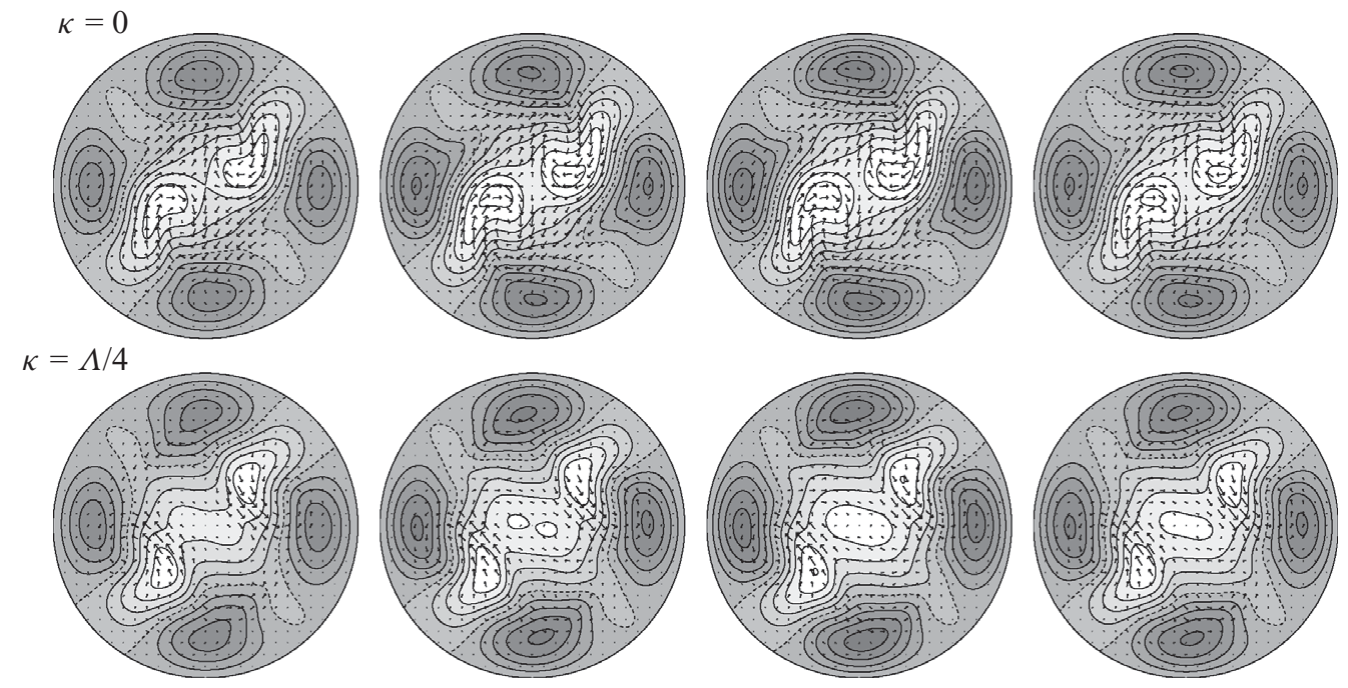

FiguRE 7. From left to right, $t=50,125,175$ and 225 snapshots of the $(\kappa, R e)=(1.52,1600)$ modulated wave, as indicated by the vertical lines in figure $6(b)$. Shown are the $z=0$ (top) and $z=\Lambda / 4$ (bottom) cross-sections with axial velocity contour lines equispaced in intervals of $\Delta u_{z}=0.1 U$. To avoid drift due to streamwise advection, snapshots are taken in a comoving frame travelling at the instantaneous advection speed from figure $6(b)$.
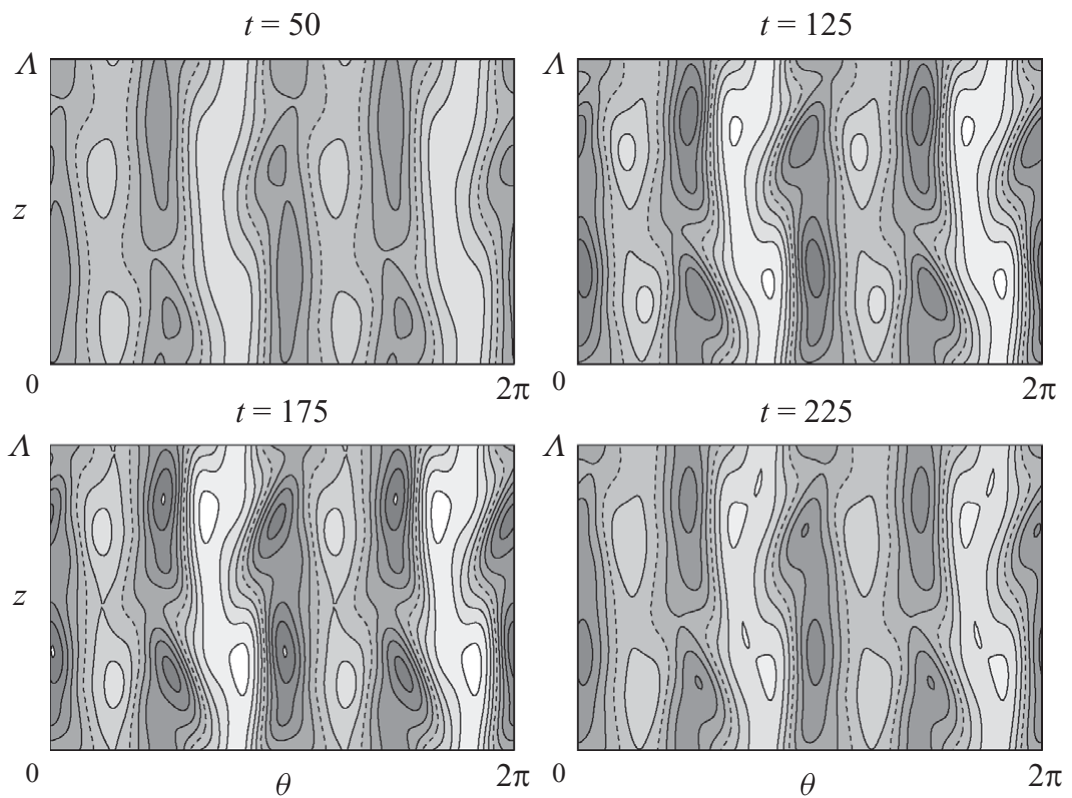

FiguRE 8 . Radial sections at $r=0.65$ of the $(\kappa, R e)=(1.52,1600)$ modulated wave. Snapshots are taken in a reference frame moving at the wave speed. Radial velocity contour lines are equispaced with intervals of $\Delta u_{r}=0.0072 U$. Regions where the flow moves towards the walls or the pipe centre are shaded dark and light, respectively.

by the laminar flow and the stable modulated wave, respectively, we have numerically computed this object with sufficient accuracy. It happens to be a long-period unstable modulated wave with a single real multiplier outside of the unit circle. 
(a)

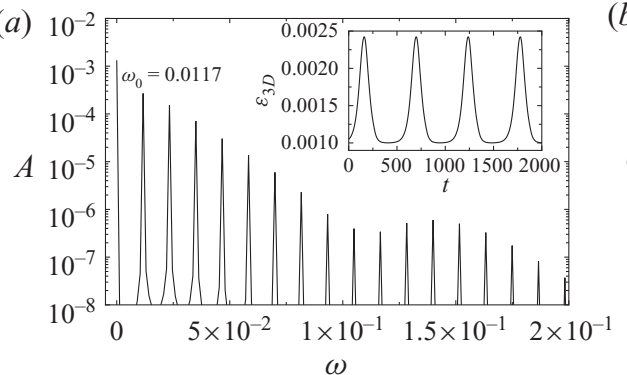

(b)

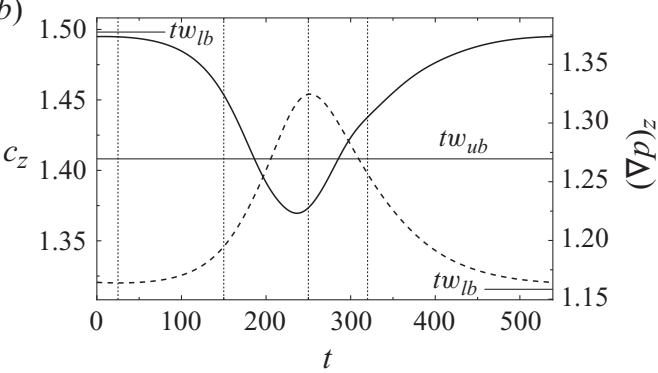

FIGURE 9. (a) Fourier transform of the non-axisymmetric streamwise-dependent modal energy contents $\left(\varepsilon_{3 D}\right)$ time stamp for the $(\kappa, R e)=(1.52,1600)$ unstable modulated wave. The time signal is plotted in the inset. $(b)$ Advection speed $\left(c_{z}\right.$, solid line) and mean axial pressure gradient normalized by its laminar value $\left((\nabla p)_{z}\right.$, dashed line) along a full period. Values for the $(\kappa, R e)=(1.52,1600)$ upper-branch $\left(t w_{u b}\right)$ and lower-branch $\left(t w_{l b}\right)$ travelling waves are indicated by horizontal lines.

The Fourier transform of the energy signal (figure $9 a$ ) reveals a period $T=538.8$, much longer than that of the stable modulated wave. The fact that the spectrum is coarsely resolved is a consequence of the limited number of periods over which the solution can be computed. Time evolution cannot produce long time-series due to the instability of the solution. Edge-tracking can potentially produce long signals, but only at the cost of extremely long computational time and with vast requirements of computational power.

Analysing the time signal itself (see the inset in figure $9 a$ ) it is clear that the solution evolves slowly for most of the time (plateau) with quick excursions to higher energies. The slow evolution could be revealing a periodic approach to a travellingwave solution. This is substantiated by figure $9(b)$, where the plateau of both speed and pressure signals is shown to approach lower-branch travelling-wave levels.

The rest of the time, the time series oscillate around upper-branch travelling-wave levels, as was the case for the stable modulated wave, but with larger amplitudes. It will soon become clear that the unstable wave essentially orbits around the stable wave and that the modulation amplitude is slowed down by the presence of the lower-branch travelling wave as the flow approaches this solution.

Figures 10 and 11 (see supplementary movies) strongly support this approach. In particular, the snapshot at $t=25$ is essentially indistinguishable from the lower-branch travelling wave.

The remaining snapshots along the trajectory confirm what the time signals already seemed to indicate: the unstable wave is always further away from the upper-branch travelling wave than the stable modulated wave. The extreme case is of course the snapshot at $t=25$, which represents the maximum distance from the upper-branch travelling wave.

As was the case for both travelling waves and the stable modulated wave, the unstable modulated wave also preserves the shift-reflect symmetry all along. Both modulated waves will shortly be shown to be related by a symmetry-preserving fold bifurcation, implying that they are just a node (stable wave) and a saddle (unstable wave) cycle on the same modulated waves branch.

\subsection{Re-continuation and the fold-of-cycles}

Straightforward $R e$-continuation of the $\kappa=1.52$ stable modulated waves by time evolution reveals that they bifurcate off the travelling-waves' upper branch precisely 

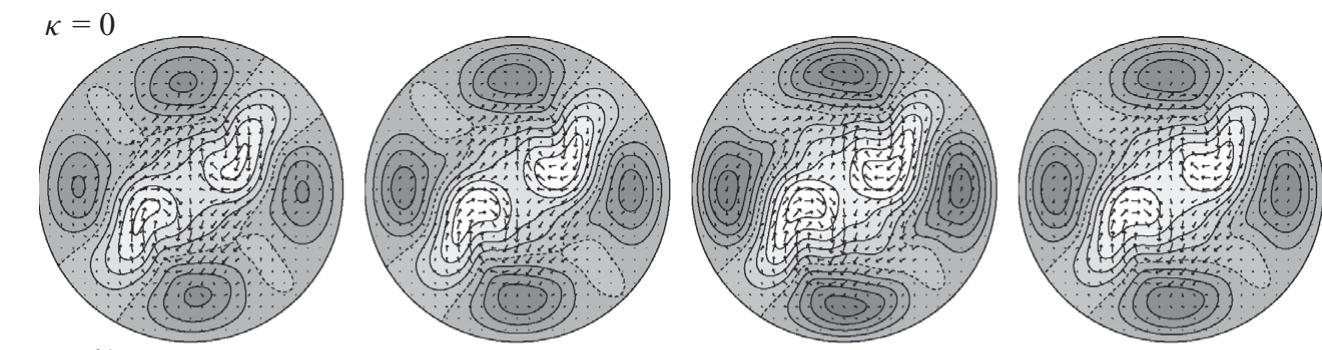

$\kappa=\Lambda / 4$
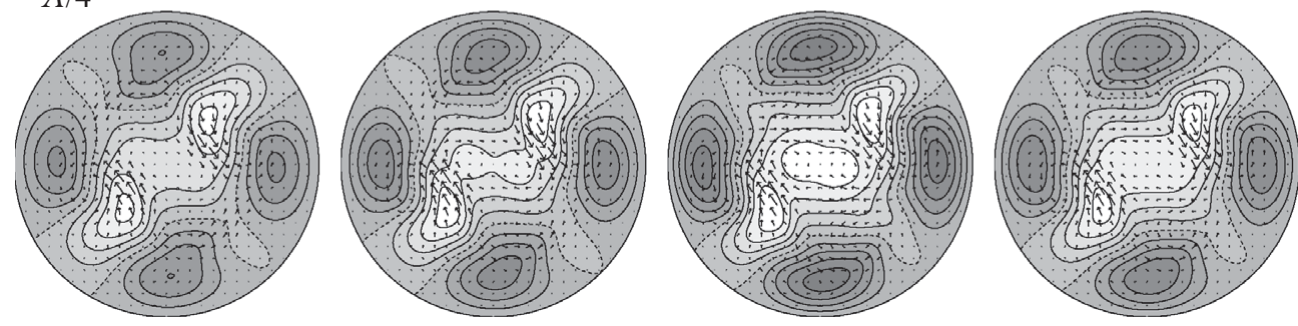

FigURE 10. From left to right, $t=25,150,250$ and 320 snapshots of the $(\kappa, R e)=(1.52,1600)$ unstable modulated wave, as indicated by the vertical lines in figure $9(b)$. Shown are the $z=0$ (top) and $z=\Lambda / 4$ (bottom) cross-sections with axial velocity contour lines equispaced in intervals of $\Delta u_{z}=0.1 U$. To avoid drift due to streamwise advection, snapshots are taken in a comoving frame travelling at the instantaneous advection speed from figure $9(b)$.
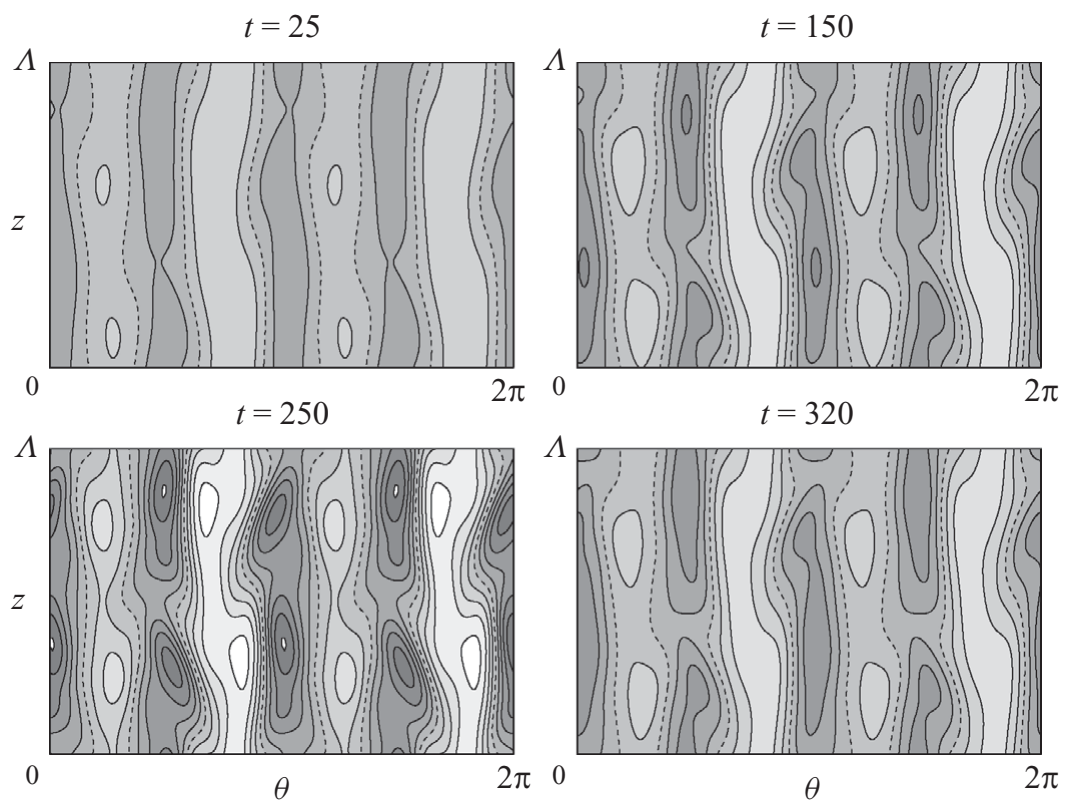

FigURE 11. Radial sections at $r=0.65$ of the $(\kappa, R e)=(1.52,1600)$ unstable modulated wave. Snapshots are taken in a reference frame moving at the wave speed. Radial velocity contour lines are equispaced with intervals of $\Delta u_{r}=0.0072 U$. Regions where the flow moves towards the walls or the pipe centre are shaded dark and light, respectively.

at the Hopf bifurcation point at $R e=1775.4$ ( $\mathrm{H}$ in figure $5 a$ ). Figure 12 shows phase map representations of the modulated waves for a discrete set of $R e$ along the branch. Two global quantities, $\varepsilon_{3 D}$ and $(\nabla p)_{z}$, have been chosen to eliminate axial drift effects. Increasing $R e$ from $R e=1600$ (towards lighter grey), the modulated wave (solid 


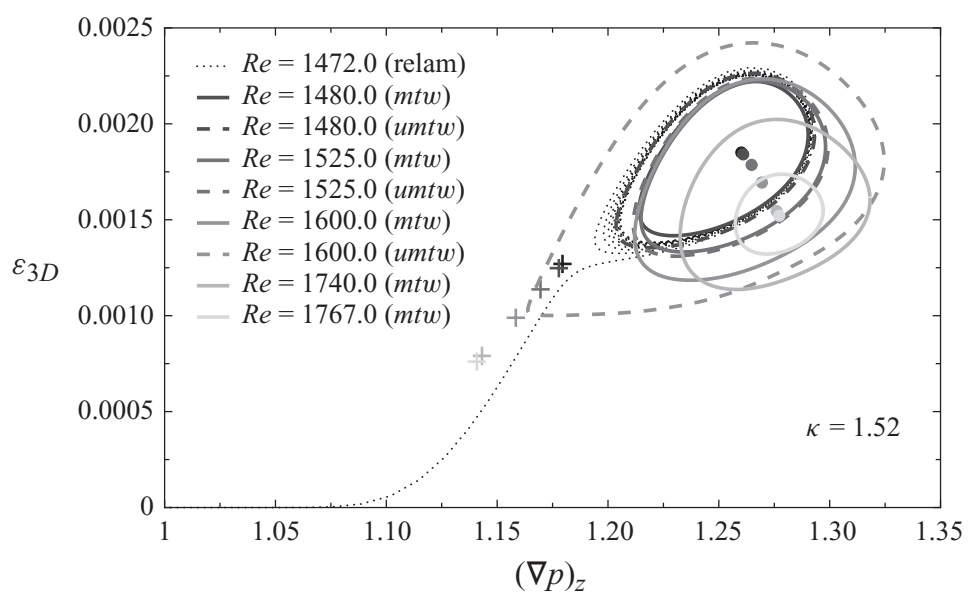

FIGURE 12. Phase map projections of travelling and modulated waves along an $R e$ continuation for $\kappa=1.52$. The axes represent non-axisymmetric streamwise-dependent energy $\left(\varepsilon_{3 D}\right)$ against mean axial pressure gradient $\left((\nabla p)_{z}\right)$. Different grey intensities correspond to different $R e$ as explained in the legend. Lower-branch and upper-branch travelling waves are marked by pluses and filled circles, respectively; modulated waves by solid (stable, $m t w$ ) and dashed (unstable, umtw) lines and escaping trajectories by dotted lines (relam).

closed loops) shrinks and closes on the upper-branch travelling wave (filled circles), to end up merging at the Hopf point $(R e=1775.4$, not shown). In the opposite direction, the wave also starts shrinking but the process stops, slightly reverses and track of the wave is lost, as pointed out by the diverging dotted trajectory. This trajectory wanders around a ghost of the disappeared modulated wave for some time (a region where dynamics are slowed down by the forthcoming hyperbolic point), then spirals away until it comes close to the lower-branch travelling wave (plus signs) and leaves the region towards the laminar flow. A close analysis seems to indicate that the branch bends and turns around a fold (saddle-node) of cycles picking up an unstable direction. In a fold-of-cycles bifurcation, a saddle and a nodal periodic orbit are created simultaneously in the same way as a saddle and a nodal equilibrium are created in a saddle-node bifurcation.

To test the advent of a fold-of-cycles bifurcation, and, at the same time, compute its precise location in parameter space, we have used the original time series that converged onto the stable modulated waves and inferred from them the least unstable Floquet multiplier. To do so, we have chosen a couple of random expansion coefficients $\left(a_{1}=\left|a_{010}^{(2)}\right|\right.$ and $\left.a_{2}=\left|a_{110}^{(2)}\right|\right)$ and taken their modulus to suppress drift dependence. Defining a Poincare section with one of them $\left(a_{2}\right)$, and recording for the other the discrete series of crossings $\left(a_{1}(q)\right)$, provides a representation of the dynamical evolution of the map associated with the Poincare application. In the vicinity of the converged modulated wave, represented by a fixed point of the Poincare application $\left(a_{1}^{m t w}\right)$, and after a time long enough that stabler directions have been purged, the dynamics are dominated by the least unstable direction and $a_{1}$ evolves following

$$
\epsilon(q)=a_{1}(q)-a_{1}^{m t w}=\epsilon(0) \mu^{q},
$$

where $\epsilon$ is a measure of the distance from the solution, varying with the discrete time $q$, and $\mu<1$ is the least unstable Floquet multiplier. A power-law fit to the computational data should produce $\mu$, provided that $a_{1}^{m t w}$ is known. Unfortunately, 

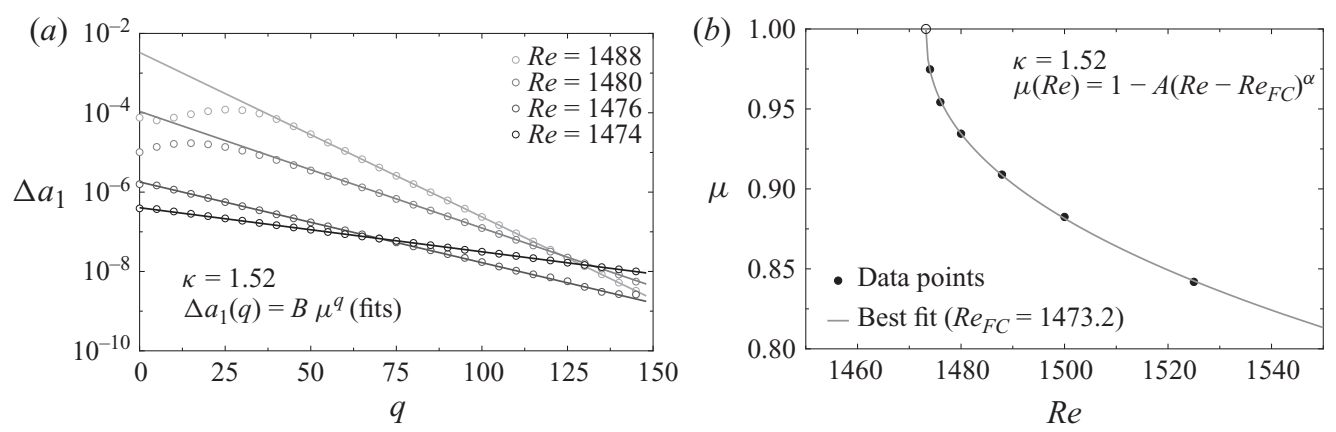

FIGURE 13. Determination of the fold-of-cycles for $\kappa=1.52$. (a) Evolution of an expansion coefficient on a Poincaré section (see text for details) for various $R e$. The fits provide the least unstable multiplier $(\mu)$ as a function of $R e .(b) \mu(R e)$ as obtained from the previous fits (dots). A suitable additional fit to the points with the functional form for $R e_{F C}$ given in the figure yields the continuous line.

$a_{1}^{m t w}$ is only reached for $q$ tending to infinity. We could be content to take $a_{1}^{m t w}$ once machine precision has been achieved, but this can take extremely long as $\mu$ approaches one. Alternatively, we can fit a power law to

$$
\Delta a_{1}(q)=a_{1}(q+1)-a_{1}(q)=\epsilon(0)(\mu-1) \mu^{q}
$$

and extract $\mu$ safely as long as the solution is reasonably approached within the region dominated by the linear regime.

Figure 13(a) shows the discrete-time evolution of $\Delta a_{1}$ on a Poincaré section defined by $a_{2}=0.045$ for several $R e$ at $\kappa=1.52$. After initial transients, the modulated wave is approached following an increasingly clear power law. As $R e$ is reduced towards where track of waves is lost, convergence becomes slower, thus indicating that the least-unstable Floquet multiplier is approaching the unit circle. The fact that the curves do not exhibit oscillations means that the multiplier is real positive, discarding flip (real negative) or Neimark-Sacker (complex pair) bifurcations. Straightforward fitting yields the Floquet multiplier, which has been plotted as a function of $R e$ in figure 13(b). The multiplier effectively approaches the unit circle as $R e$ decreases, and it does so with increasing slope. It is now justified to assume that a fold bifurcation is being approached and that the slope must go to infinity (we are plotting against the parameter in a turning point). This allows us to introduce a suitable fitting law $\left(\mu(R e)=1-A\left(R e-R e_{F C}\right)^{\alpha}\right)$ and retrieve from it the critical value at which the fold-of-cycles takes place $\left(R e_{F C}\right)$.

This procedure has been repeated for every $\kappa$ at which track of the modulatedwaves branch was lost. They all follow the trend exemplified for $\kappa=1.52$ and, as a result, a fold-of-cycles bifurcation curve $\left(\operatorname{Re}_{F C}(\kappa)\right)$ can be determined.

Unstable modulated waves (dashed lines in figure 12) fit nicely into the description just portrayed. They populate the branch of waves once it has become unstable in the fold-of-cycles.

As $R e$ increases from the fold-of-cycles point, the nodal branch approaches the upper branch of travelling waves until eventual collision at the Hopf point, while the saddle branch grows larger and larger. It is argued later that in the absence of exotic symmetry-breaking events, the unstable modulated wave ends up colliding with the lower branch of travelling waves in a saddle-loop bifurcation, where it ceases to exist without any other consequence. In a saddle-loop bifurcation, the solution slows 

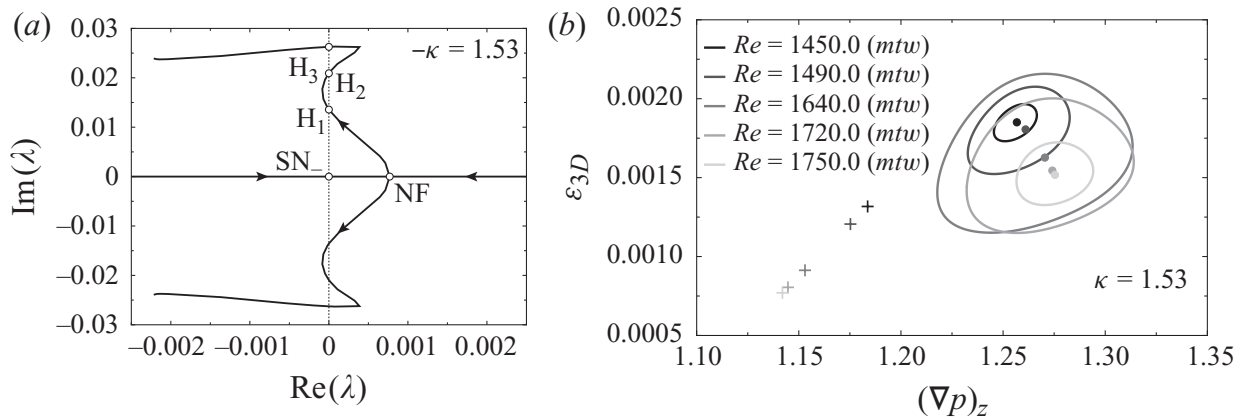

Figure 14. (a) Eigenvalue trajectories for $\kappa=1.53$. $(b)$ Phase map projections of travelling and modulated waves along an $R e$-continuation for $\kappa=1.53$. See the caption of figure 12 for explanations.

down in a certain region of the cycle as it approaches an hyperbolic saddle and finally becomes an infinite-period orbit homoclinic to the saddle before disappearing. Endorsing this scenario is the fact that unstable modulated waves not only grow large, but also get closer and closer to lower-branch travelling waves as $R e$ increases (see plus signs and dashed loops in figure 12) and clearly become pointed as this happens.

As already mentioned, decreasing $R e$ causes both branches to merge in a fold-ofcycles, leaving unstable travelling waves as the only existing solutions. Any trajectory issued from the vicinity of the upper-branch wave spirals away, temporarily orbits around the ghost of the disappeared modulated wave, and then continues spiralling away until the trajectory manages to take a detour around the lower-branch travelling wave and finally leaves towards the basic laminar profile $\left(\varepsilon_{3 D},(\nabla p)_{z}\right)=(0,1)$ along the only unstable direction of the travelling wave.

Continuation in the geometrical parameter $\kappa$ causes the node-focus transition point to move around without noticeable impact on the eigenvalues $R e$-trajectories, as can be concluded from figures $3(c, d)$ and $5(a)$. It becomes clear that for wavenumbers slightly beyond $\kappa \gtrsim 1.52$, the trajectories will experience an additional couple of zerocrossings, adding a pair of Hopf bifurcation points to the already existing one. This is illustrated for $\kappa=1.53$ in figure 14(a), where the successive crossings along the upper branch have been labelled $\mathrm{H}_{1}, \mathrm{H}_{2}$ and $\mathrm{H}_{3}$ as $R e$ is increased. Continuation of the modulated waves branch for $\kappa=1.53$, shown in figure $14(b)$, reveals that they connect supercritically to the upper branch of travelling waves in both directions at the two Hopf points that are at a larger distance from the saddle-node bifurcation, i.e. $\mathrm{H}_{2}$ and $\mathrm{H}_{3}$ in figure 14(a). The fold-of-cycles that was present at $\kappa=1.52$ is no longer present at $\kappa=1.53$. It is to be expected that unstable modulated waves exist all the same, except that they are issued from a fold-of-cycles of a second branch of supercritical modulated waves bifurcating at lower $R e$ or directly created at a subcritical Hopf of upper-branch travelling waves (see discussion).

We shall extend this notation, $\mathrm{H}_{1}, \mathrm{H}_{2}$ and $\mathrm{H}_{3}$, to denote the three potential Hopf crossings for any $\kappa$ in the range studied. It is clear that for decreasing $\kappa, \mathrm{H}_{1}$ and $\mathrm{H}_{2}$ approach, collide and disappear, leaving $\mathrm{H}_{3}$ alone. Instead, for increasing $\kappa$ it is $\mathrm{H}_{2}$ and $\mathrm{H}_{3}$ that approach and collide, leaving $\mathrm{H}_{1}$ alone as long as the node-focus point remains on the right side of the complex plane. 


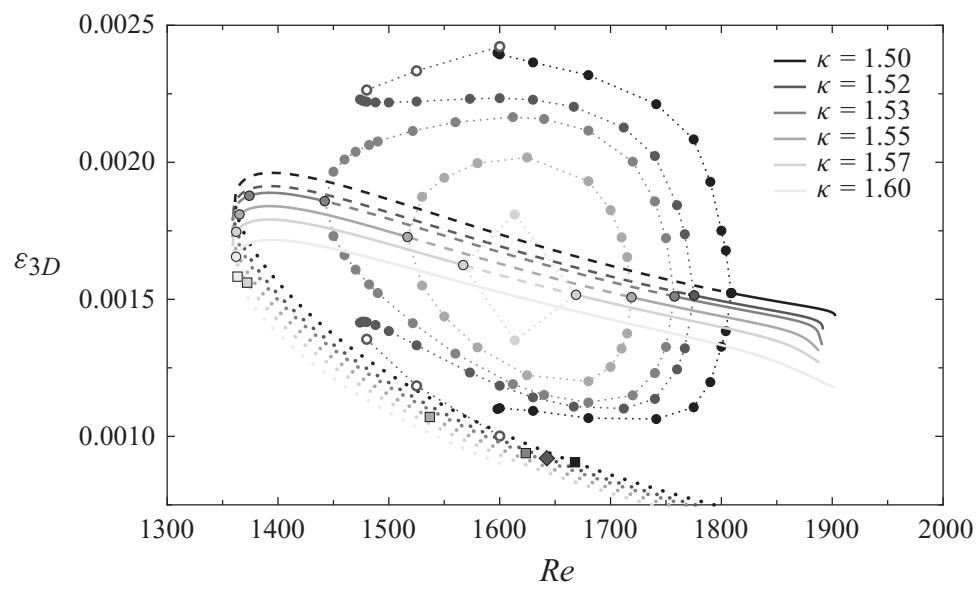

FIGURE 15. Non-axisymmetric streamwise-dependent energy $\left(\varepsilon_{3 D}\right)$ as a function of $R e$ and $\kappa$ (different grey intensities as indicated in the legend). Lines represent travelling waves: solid, dotted and dashed for 0,1 and 2 unstable directions. Filled circles on the curves represent Hopf points. The remaining circles represent modulated waves (filled for stable, empty for unstable at $\kappa=1.52$ ). Saddle-loop bifurcations are indicated by squares (a diamond for $\kappa=1.52$ ) on the lower branches.

Adding the Re-dependence helps to clarify the situation. This is done in figure 15, where $\varepsilon_{3 D}$ has been plotted as a function of $R e$ and $\kappa$ (varying grey intensity as indicated by the legend) for both travelling waves (solid, dotted and dashed thick lines for zero-, one- and two-dimensional unstable manifolds) and modulated waves (circles indicating minimum and maximum values of $\varepsilon_{3 D}$ along a period; filled for stable, empty for unstable waves). Lower-branch travelling waves have a single unstable direction. For all cases shown, as they turn around the saddle-node point, a second unstable direction appears. Low- $\kappa($ e.g. $\kappa=1.52)$ waves remain unstable until they regain stability at Hopf bifurcations, whence supercritical branches of stable modulated waves are issued, as already discussed for figure 12. Traces of the turning point that we identified with a fold-of-cycles are clear from the bending tips of the modulated wave branches, and from the converging trend of stable and unstable branches (shown for $\kappa=1.52$ ). High- $\kappa($ e.g. $\kappa=1.60)$ waves regain stability almost immediately at Hopf bifurcations but no stable branch of waves could be detected. Intermediate- $\kappa$ (e.g. $\kappa=1.53)$ waves exhibit a mixed behaviour by regaining stability early on the upper branch, but losing it again temporarily for some range of Re. The whole intervening unstable range is nevertheless covered by a stable branch of modulated waves connecting both Hopf points, as illustrated in figure 14(b). At even higher $\kappa$ (not shown), travelling waves become stable at the saddle-node, as was the case for $\kappa=1.70$ of figure $3(b, d)$. Returning to upper-branch travelling waves that were unstable at onset, the branch of modulated waves that must be born at their early stabilization could not be detected with time evolution. This is a clear sign that they may bifurcate subcritically, with the branch pointing in the increasing Re direction.

Figure 16 shows the angular frequency $\omega$ of the modulated waves as a function of $R e$ and $\kappa$. Only a subset of $\kappa$ has been plotted for clarity, and, for most of the wavenumbers, only as long as the branch is stable, i.e. accessible through time evolution. Along with the waves, the locus of Hopf bifurcation points (enveloping line) has been added by plotting the imaginary part of the bifurcating eigenvalues. Along this curve, $\kappa$ varies continuously but not monotonically. The curve has been 


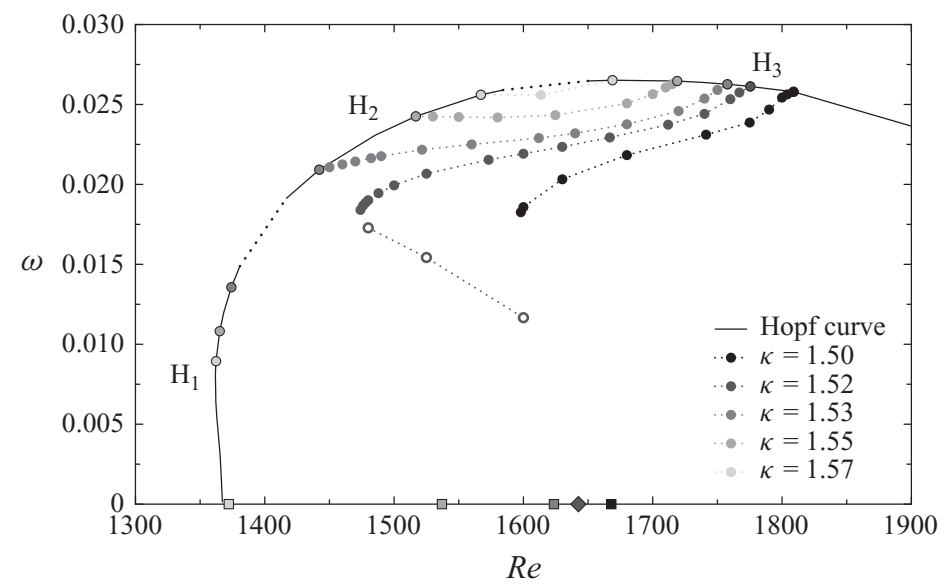

Figure 16. Angular frequency $(\omega)$ of stable (filled circles) and unstable (empty circles, only for $\kappa=1.52$ ) modulated waves. The enveloping line represents the imaginary part of the bifurcating eigenvalues along the locus of Hopf bifurcation points (filled circles on the line). The three segments, labelled $\mathrm{H}_{1}, \mathrm{H}_{2}$ and $\mathrm{H}_{3}$, result from splitting the Hopf curve at its two tangencies with iso- $\kappa$ lines. Saddle-loop bifurcation points are indicated by squares (a diamond for $\kappa=1.52$ ) on the $\omega=0$ axis.

split into three monotonical segments corresponding to up to three independent zero-crossings that the complex pair of eigenvalues might undergo $\left(\mathrm{H}_{1}, \mathrm{H}_{2}\right.$ and $\left.\mathrm{H}_{3}\right)$. Naturally, the modulational frequency of the bifurcating modulated waves is dictated by the imaginary part of the eigenvalues at the Hopf bifurcation. This figure strongly endorses the fold-of-cycles hypothesis for the non-reconnecting branches, especially for $\kappa=1.52$, for which a few unstable modulated waves (empty circles) have been computed. The decreasing frequency around the fold-of-cycles is suggestive of an eventual saddle-loop collision with lower-branch travelling waves, which demands a vanishing frequency (period going to infinity) as the modulated wave transforms into a non-robust homoclinic loop and disappears.

It is clear that for $1.52 \lesssim \kappa \lesssim 1.53$ a change of behaviour takes place. Modulated wave branches go from reconnecting to the Hopf curve to bending and losing stability. It seems therefore reasonable that for some intermediate value, a branch of solutions may become tangent to the Hopf curve, precisely at the $\kappa$-value for which the couple of additional eigenvalue crossings start occurring, i.e. where $\mathrm{H}_{1}$ and $\mathrm{H}_{2}$ merge. Slightly above this value, the curve would split into two. One of the segments connects supercritically two of the three existing Hopf points $\left(\mathrm{H}_{2}\right.$ and $\left.\mathrm{H}_{3}\right)$. The other segment would be issued from the third Hopf point $\left(\mathrm{H}_{1}\right)$ and, for purely geometrical reasons, it would have to do so supercritically towards lower $R e$. Unfortunately, the room left in parameter space for the stable branch of waves before it bends back and becomes unstable is tiny and very close to the Hopf bifurcation line where the relevant complex pair of eigenvalues exhibits a vanishing real part. As a consequence, transients are far too long to allow computation of the stable modulated waves in a feasible time scale. This ultimately renders the numerical determination of the borders of existence of such solutions within this region extremely difficult. For even higher $\kappa$, but not exceeding the value for which upper-branch travelling waves are stable at onset, modulated waves can only bifurcate subcritically from $\mathrm{H}_{1}$ towards increasing $R e$ and with vanishing frequencies, as required by the double-zero bifurcation scenario that takes place. 
(a)

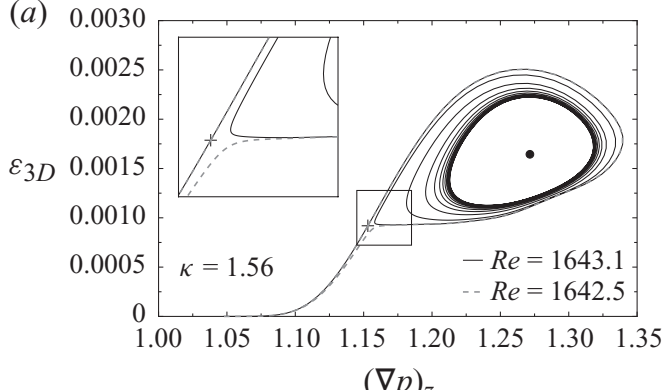

(b)

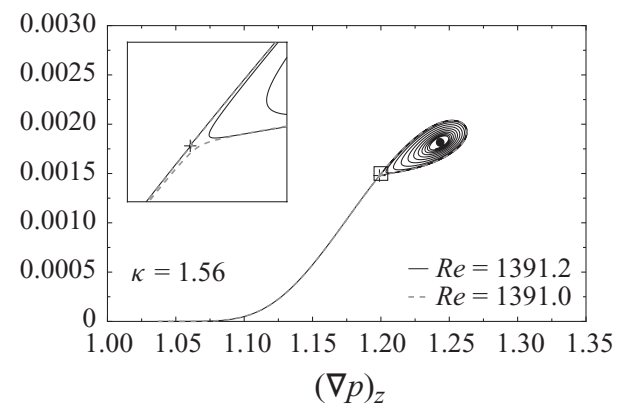

FiguRE 17. Saddle-loop bifurcation at $(a) \kappa=1.52$ and $(b) \kappa=1.56$. Trajectories issued from the lower-branch travelling wave (plus sign), for Re just below (grey dashed line) and just above (black solid line) the global bifurcation. The black filled circle shows the location of the upper-branch travelling wave. The boxed region is enlarged in the inset.

\subsection{The saddle-loop bifurcation}

The surmised saddle-loop bifurcation of unstable modulated waves as they collide with lower-branch travelling waves can be easily bound to any desired accuracy. For $R e$ smaller than where this global bifurcation takes place, the attractor (either the upper-branch travelling wave or the stable modulated wave) is shielded by an unstable cycle (unstable modulated wave) that prevents trajectories issued from the saddle (lower-branch travelling wave) from converging onto it. This is exactly what led in $\S 3.2$ to the computation of unstable modulated waves. In the event of a saddle-loop bifurcation, the unstable cycle would collide with the saddle generating a non-robust homoclinic connection and disappear thereafter. This critically changes the phase map structure, rendering the attractor reachable by trajectories issued from the saddle. By tracking this change in behaviour we can pinpoint the precise parameter values at which the bifurcation occurs.

Figure $17(a, b)$ shows trajectories issued from the lower-branch travelling wave (plus/minus a small random perturbation) for $\kappa=1.52$ and $\kappa=1.56$, at $R e$-values bracketing the saddle-loop bifurcation. Some (uninteresting) trajectories relaminarize uneventfully, while their counterparts (perturbed in the opposite direction), loop around the upper-branch travelling wave and return onto the lower-branch wave. At this point, the lower-Re trajectory (dashed grey curve) bends downwards and becomes laminar, while the higher-Re one bends upwards and spirals around the upper-branch wave (see insets). The tighter the bracketing, the closer the bounding trajectories approach the lower-branch wave before parting. At the limit where the bifurcation takes place $\left(R e_{S L}\right)$, trajectories would follow a homoclinic connection.

The difference between figures $17(a)$ and $17(b)$ resides in the behaviour of the trajectories above $R e_{S L}$. For $\kappa=1.56$, the trajectory converges onto the stable upperbranch wave. For $\kappa=1.52$, the upper-branch travelling wave is unstable and a stable modulated wave is reached instead.

Tracking this global bifurcation systematically for varying $\kappa$ yields a saddle-loop bifurcation curve, parametrized by $\operatorname{Re}_{S L}(\kappa)$.

The location of the saddle-loop point for $\kappa=1.52$ in figures 15 and 16 (dark grey filled diamond), relative to the unstable modulated waves branch provides strong evidence that these are the waves that undergo the global bifurcation. Even if the unstable modulated wave frequency is still far from vanishing at $R e=1600$ (last 
computed point), Gaspard (1990) pointed out that the period of a cycle approaching an hyperbolic fixed point diverges as

$$
T=\frac{2 \pi}{\omega} \sim-\lambda^{-1} \log \left(R e_{S L}-R e\right)+d,
$$

where the factor $\lambda$ is the eigenvalue of the unstable direction of the hyperbolic fixed point, $R e_{S L}$ is the parameter value at the global bifurcation, $d$ is a constant, and $\log$ represents the natural logarithm. As a consequence, $\omega$ sinks very fast only in the vicinity of the bifurcation. Unfortunately, it is extremely difficult to test this law in the case at hand for lack of computed unstable waves close enough to the global bifurcation. Their computation becomes extremely difficult as unstable multipliers escalate due to the diverging period. Nevertheless, even if the period is still not dramatically diverging at $R e=1600$, figure 12 clearly reveals a tendency of the unstable modulated wave to conform to the pointed trajectory shape that later $\left(R_{S L} \sim 1643\right)$ becomes an orbit homoclinic to the saddle (figure 17a) before disappearing.

As a by-product of tracking this global bifurcation, it should be noted that none of the random perturbations to the lower-branch travelling wave used to initiate time-integration ever produced chaotic dynamics, the shift-reflect symmetry being naturally restored by the system in all cases. This discards the occurrence, at least within the explored parameter region, of homoclinic tangencies that could be expected generically in the case where the symmetry happened to be disrupted.

\subsection{Unfolding of the Takens-Bogdanov bifurcation}

The twofold azimuthally-periodic, shift-reflect symmetric family of travelling waves has been continued both in $R e$ and $\kappa$, and a systematic linear-stability analysis has been carried out. In this manner, the locus of the saddle-node bifurcation between the upper- and lower-branch travelling waves, and that of the Hopf bifurcation of upperbranch travelling waves has been tracked in parameter space. Results are summarized in figure 18. To the left of the saddle-node bifurcation lines $\left(\mathrm{SN}_{+}\right.$and $\left.\mathrm{SN}_{-}\right)$, there exist no travelling waves. These appear in saddle-node bifurcations to the right of the saddle-node lines. Upper-branch waves produced in $\mathrm{SN}_{+}$are stable nodes, whereas those appearing in $\mathrm{SN}_{-}$are unstable (see figure 3), all lower-branch travelling waves being unstable with a single unstable direction. Almost immediately after springing into existence, upper-branch travelling waves experience a node-focus transition (NF of figure 3). This non-bifurcation curve is not shown in the figure, as it is extremely close to the saddle-node curve. $\mathrm{SN}_{+}, \mathrm{SN}_{-}$and $\mathrm{NF}$ emanate from a Takens-Bogdanov or double-zero bifurcation point (TB). At this point, two real eigenvalues collide exactly at the origin of the complex plane and become a conjugate pair. From this same point, a Hopf bifurcation line $(\mathrm{H})$ is issued downwards to the right of $\mathrm{SN}_{-}$.

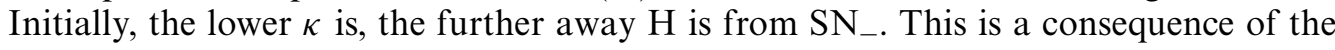
left-opening parabolic shape of the complex eigenvalues (figure 3). As $\kappa$ is reduced, NF moves away from the origin of the complex plane and the distance along the trajectory, which is related to Re, before crossing gets longer. Further along $\mathrm{H}$, a couple of successive upward and downward bendings occur. The three segments into which they split the curve can be identified with $\mathrm{H}_{1}, \mathrm{H}_{2}$ and $\mathrm{H}_{3}$, the three potential crossings the eigenvalues may undergo at any given $\kappa$. Above this line, upper-branch travelling waves are stable. Below it they are always unstable. As a consequence of its last downward turn, for sufficiently large $R e$ all upper-branch solutions are left stable as far as this bifurcation diagram is concerned and no secondary instabilities 


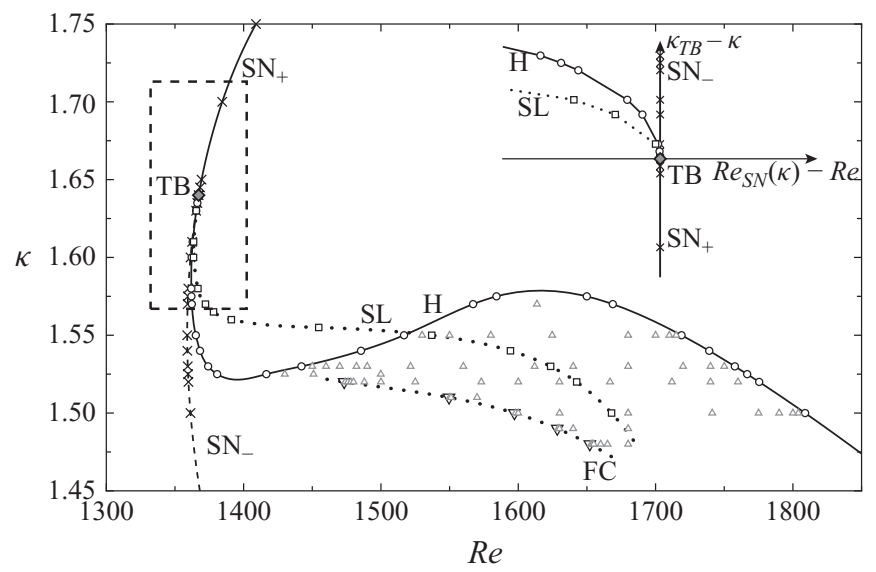

FIGURE 18. Bifurcation curves in the $(\kappa, R e)$-parameter space. $\mathrm{SN}_{+}$(solid line with crosses) and $\mathrm{SN}_{-}$(dashed line with crosses) represent stable and unstable saddle-node bifurcations of travelling waves. $\mathrm{H}$ (solid line with circles) is the Hopf bifurcation curve of upper-branch travelling waves. TB is the Takens-Bogdanov (double-zero) bifurcation point (grey diamond). Stable modulated waves are indicated by triangles, with FC (dotted line with black down-pointing triangles) representing a fold-of-cycles. The saddle-loop curve (SL) is indicated by squares joined by a dotted line. The inset shows a transformed zoom of the dashed region, with new coordinate axes $\kappa_{T B}-\kappa$ and $R e_{S N}(\kappa)-R e$.

take place. To complete the phenomenological description, modulated waves have also been added into the picture (empty triangles). They occupy a region enclosed between the Hopf line and another line defined as the locus of turning points where modulated waves cease to exist. This line is a bifurcation curve corresponding to a fold-of-cycles, whereby stable modulated waves turn around and become unstable.

Also issued from $\mathrm{TB}$ to the right of $\mathrm{H}$, and running on the lower branch of travelling waves, a global bifurcation curve has been identified. It is the locus of saddle-loop bifurcations, where unstable modulated waves, created either at a subcritical Hopf or at the fold-of-cycles, collide with lower-branch waves and disappear.

\section{Discussion}

It is at this point convenient to define an alternative couple of parameters: $\kappa_{T B}-\kappa$ and $\operatorname{Re}_{S N}(\kappa)-R e$, where $\kappa_{T B}$ is the value of $\kappa$ at the Takens-Bogdanov point and $\operatorname{Re}_{S N}(\kappa)$ is the value of $R e$ at the saddle-node point for each given $\kappa$. The transformation has been applied to the region indicated by the dashed square in figure 18 and plotted in the inset. The resulting bifurcation diagram is reminiscent of the double-zero bifurcation described by Wiggins (2003). Of the two equivalent normal forms proposed for this bifurcation by Takens (1974) and Bogdanov (1975), the former can be applied to the present bifurcation by simply taking $\mu_{1} \simeq \kappa_{T B}-\kappa$ and $\mu_{2} \simeq R e_{S N}(\kappa)-R e$ in Wiggins (2003):

$$
\left.\begin{array}{l}
\dot{x}=y, \\
\dot{y}=\mu_{1}+\mu_{2} y+x^{2}+b x y,
\end{array}\right\}
$$

with $b=+1$. A detailed analysis of the alternative normal form can be found in Kuznetsov (1995). 
This second-order normal form provides an accurate description of all the phenomenology observed in the immediate vicinity of the Takens-Bogdanov point. From the Takens-Bogdanov point (inset of figure 18), stable $\left(\mathrm{SN}_{+}\right)$and unstable $\left(\mathrm{SN}_{-}\right)$saddle-node lines extend along the vertical axis downwards and upwards, respectively. Issued from the same point is a subcritical Hopf curve with a left-opening half-parabolic shape, to the left of the unstable saddle-node line. The unstable node created at the saddle-node immediately becomes a focus and later recovers stability upon crossing the Hopf curve leftwards. An unstable branch of unstable periodic orbits originates surrounding the stabilized equilibrium. A close analysis of the bifurcation reveals that the unstable periodic orbits grow large and collide with the unstable saddle in a saddle-loop homoclinic bifurcation. The unstable periodic orbits disappear with no consequence for the saddle equilibrium, leaving it, together with the stable focus, as the only surviving solutions. The detected saddle-loop curve, also branching off the Takens-Bogdanov point, is thus accounted for. This entails that the Hopf points that are closest to the saddle-nodes in figure 15 and on $\mathrm{H}_{1}$ in figure 16 (circles), are connected to the corresponding saddle-loop points (squares) via a branch of modulated waves. This branch, as dictated by the Takens-Bogdanov scenario, must be subcritical close enough to TB.

An analysis of the second-order normal form is nevertheless insufficient to account for the existence of stable modulated waves and the back and forth bending of the Hopf curve. It is however possible to analyse in some detail what the effect of higherorder terms would be if the conditions for a non-degenerate double-zero bifurcation were nearly violated and higher-order terms were not negligible some distance away from the bifurcation point. A complete analysis of the codimension-three bifurcation resulting from a vanishing second-order coefficient ( $b$ in (4.1)) can be found in Dumortier et al. (1991). It is unlikely, unless some symmetry at play cancels exactly some second-order coefficients, which is not the case (the eigenfunctions involved preserve all symmetries of the solution), that simple tuning of two parameters will cross a codimension-three point. Therefore, it is not the codimension-three point we are interested in, but a perturbation around it that renders higher-order terms important at some distance from the codimension-two point under analysis.

Such an analysis was carried out by Barkley (1990) when studying similar phenomena in plane Poiseuille flow, i.e. the appearance of modulated waves in channel flow. Previous studies (Pugh \& Saffman 1988; Soibelman \& Meiron 1991) had reported the onset of a modulational instability of travelling waves when the flow was driven by a constant pressure gradient. No instability was detected for the same waves when the flow was driven at constant mass flux. Introducing a new parameter allowing the connection continuously of both possible boundary conditions, a bifurcating scenario arose as a natural explanation. It was suggested that while a double-zero bifurcation could explain the appearance of unstable modulated waves, the simple inclusion of third-order terms in the second-order normal form could explain the existence of stable modulated waves for some parameter range. According to the normal form theory, the extended normal form needs only the inclusion of a couple of third-order terms (Guckenheimer \& Holmes 1983; Knobloch 1986; Iooss \& Adelmeyer 1998)

$$
\left.\begin{array}{l}
\dot{x}=y, \\
\dot{y}=\mu_{1}+\mu_{2} y+x^{2}+k_{1} x y+k_{2} x^{3}+k_{3} x^{2} y,
\end{array}\right\}
$$

where appropriate scaling can be performed such that $k_{3}= \pm 1$. The second-order coefficient $k_{1}$ is required to be sufficiently small so that third-order terms come 
into play, but non-zero so that non-degeneracy of the regular Takens-Bogdanov is preserved for sufficiently small $\mu_{1}$ and $\mu_{2}$.

We shall not reproduce here the analysis of the third-order-extended normal form for the Takens-Bogdanov bifurcation. Instead, we refer the reader to Barkley (1990) for a detailed unfolding, and pick up on the main results that are relevant to the present case. It can be shown that appropriate tuning of the coefficients enables this normal form to capture some of the phenomenology observed but so far unexplained. For example, setting $k_{3}=+1$ and $k_{1}>0$ gives the right eigenvalue path up to the first right turn after the node-focus transition (figures $3 c, d$ and $5 a$ ). As a consequence, the Hopf curve experiences a first turning point as seen in figure 18, allowing for multiplicity of Hopf points at any given $\kappa$ above some minimum value. A most relevant result concerns the nature of the Hopf curve itself. It has already been mentioned that in the vicinity of the Takens-Bogdanov point, the Hopf curve happens to be subcritical in the sense that the first Lyapunov coefficient is positive and bifurcating periodic orbits are unstable. Restricting (4.2) to the Hopf curve and with an appropriate change of variables and rescaling, we obtain

$$
\left.\begin{array}{l}
\dot{\rho}=\rho\left(\beta_{1}+\beta_{2} \rho^{2}+s \rho^{4}\right)+O\left(\rho^{7}\right), \\
\dot{\phi}=1,
\end{array}\right\}
$$

with $s=+1$, which is the normal form for the degenerate Hopf or Bautin bifurcation (Kuznetsov 1995). At a Bautin point, bifurcating periodic orbits go from being stable at onset to being unstable or vice versa. $\beta_{2} \sim k_{1}-3 k_{2} \mu_{2}$ is the first Lyapunov coefficient and governs the change in criticality along the Hopf curve. Taking $k_{2}>0$ will make $\beta_{2}$ vanish for some value of $\mu_{2}$, leaving the Hopf curve supercritical beyond some point sufficiently far from the Takens-Bogdanov point. This is exactly what is needed to account for supercritical bifurcation of stable modulated waves. The Bautin bifurcation also requires that the stable modulated waves turn around in a fold-ofcycles, whenever they cannot branch to another Hopf point, becoming unstable. Since the original unstable waves collided with the saddle in a saddle-loop bifurcation in the frame of the Takens-Bogdanov bifurcation, it is to be expected that they will continue exhibiting the same behaviour even if they now initially bifurcate supercritically. Thus, even though we could only systematically follow stable modulated waves to their fold bifurcation (figure 15), the computation of a few solutions on the unstable branch at $\kappa=1.52$ and the determination of saddle-homoclinic connections, enables us to anticipate their fate: they most probably disappear in saddle-loop bifurcations. This was already justified upon explaining figure 16, where the trend of the angular frequency suggested that the period could go to infinity after turning around. This is exactly what the saddle-loop bifurcation requires for cycles approaching infiniteperiod homoclinic bifurcations.

The Bautin point must be somewhere along the Hopf curve before the first turning point. As it happens, supercriticality is only extremely mild until about the turning point (the locus of the fold-of-cycles happens to be very close to the Hopf curve), resulting in our inability to compute the stable modulated waves that must be present. This is so because close to the Hopf curve, the dynamics are extremely slow and the transients accordingly long. These are the waves that are expected to turn around, become unstable and be destroyed in saddle-loops as predicted by third-order terms and evidenced by our numerical results. Beyond the turning point, supercriticality means branching towards lower $\mu_{1}$ (higher $R e$ ). We have computed these waves, 


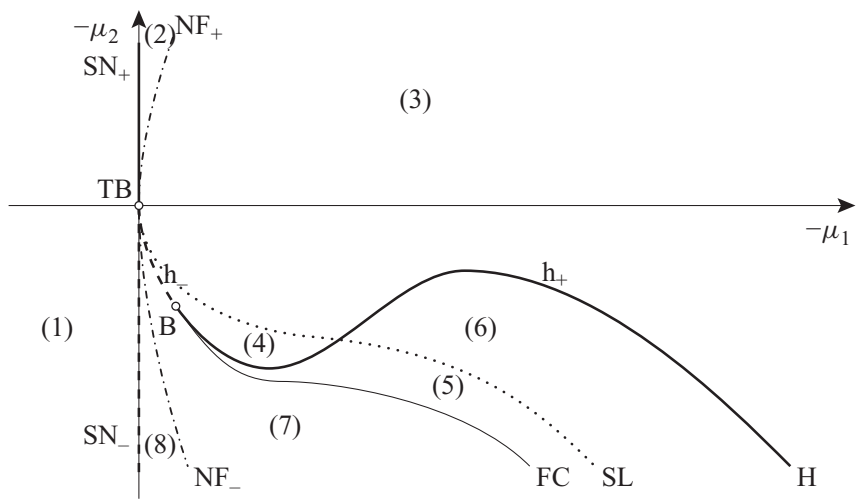

FIGURE 19. Sketch of the bifurcation diagram in the $\left(-\mu_{1},-\mu_{2}\right)$-parameter space for the normal form. Represented are the two codimension-two points TB (double-zero or Takens-Bogdanov) and B (Bautin or degenerate Hopf). B splits the Hopf curve $(\mathrm{H})$ into two segments, one subcritical $\left(h_{-}\right.$, dashed thick line), the other supercritical $\left(h_{+}\right.$, solid thick line). From TB, stable and unstable saddle-node curves $\left(\mathrm{SN}_{+}\right.$and $\left.\mathrm{SN}_{-}\right)$, the Hopf curve and a saddle-loop curve (SL, dotted line) are born, together with stable and unstable node-focus transition curves $\left(\mathrm{NF}_{+}\right.$and $\mathrm{NF}_{-}$, dash-dotted lines). A fold of cycles (FC) is issued from B. Numbers in parenthesis indicate different regions and point to the phase space flows in figure 20 .

but their fate crucially depends upon the second turning of the Hopf curve, and third-order terms tell us nothing about it.

Adding fourth-order terms is a straightforward but tedious calculation that we shall not reproduce here. It cannot be expected that fourth-order amplitude equations capture the behaviour reliably so far from the Takens-Bogdanov point, yet there is strong numerical evidence that fourth-order terms contribute. They allow for an extra turning point of the Hopf curve, without altering any of the previous predictions. As a result, stable modulated waves born between the two turning points can disappear in a second supercritical Hopf after the second turn, as illustrated in figures 15 and 18. Further down the Hopf curve, beyond the second turning point, stable modulated waves either behave just as explained or progress stably until they meet the foldof-cycles and turn around. This curve must emerge from the Hopf curve precisely at the Bautin point, the existence of which can be inferred but its location was not determined precisely with the tools at hand.

Figure 19 completes the picture just sketched in a diagram for all bifurcations in parameter space. Parameters $-\mu_{1}$ and $-\mu_{2}$ have been chosen to ease comparison with figure 18. The full diagram is organized around the Takens-Bogdanov (TB) codimension-two point. This point separates two branches of saddle-node lines $\left(\mathrm{SN}_{+}\right.$and $\left.\mathrm{SN}_{-}\right)$. At $\mathrm{SN}_{+}$, a stable node and a saddle equilibrium coalesce, while crossing $\mathrm{SN}_{-}$generates an unstable node and a saddle. The node, stable or unstable, experiences a quick transition and becomes a focus, stable or unstable, in a nodefocus transition $\left(\mathrm{NF}_{+}\right.$and $\left.\mathrm{NF}_{-}\right)$. These are non-bifurcation curves. From $\mathrm{TB}$, a Hopf bifurcation curve $\mathrm{H}$ springs quadratically to the same side as $\mathrm{SN}_{-}$. Sufficiently close to TB, periodic orbits bifurcate subcritically from the node at $\mathrm{H}\left(\mathrm{h}_{-}\right)$. Further away, $\mathrm{H}$ changes criticality at a codimension-two Bautin point (B, degenerate Hopf) and becomes supercritical $\left(h_{+}\right)$. At $B$, a fold-of-cycles curve (FC) is born to the $h_{+}$side of $\mathrm{H}$ and parting from it quadratically. Between $\mathrm{h}_{+}$and $\mathrm{FC}$, stable periodic orbits exist. At $\mathrm{h}_{-}$and $\mathrm{FC}$, unstable periodic orbits are generated. They must all cease 
$\mathrm{SN}_{+}$

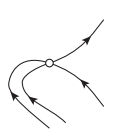

(4), $\mathrm{H}$

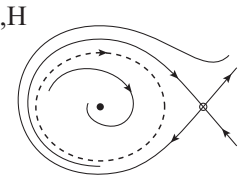

(7), $\mathrm{NF}_{-}$

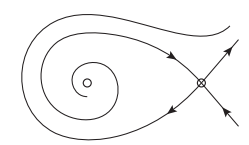

(2)

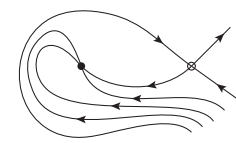

(5)

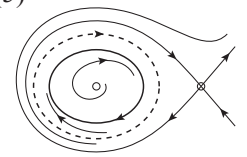

(8)

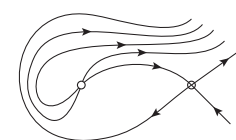

(3), $\mathrm{H}, \mathrm{NF}_{+}$

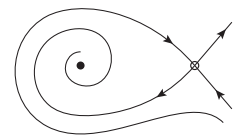

(6)

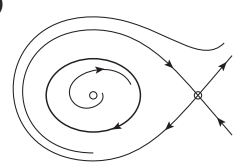

$\mathrm{SN}$

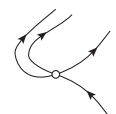

SL

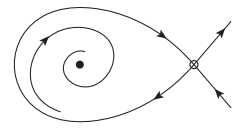

FC

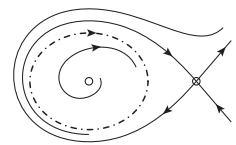

TB

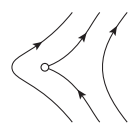

FIGURE 20. Phase map diagrams corresponding to all bifurcation curves and numbered regions in figure 19. Small circles correspond to relative equilibria (filled for stable equilibria), and closed thick lines to relative periodic orbits (solid for stable, dashed for unstable orbits). Thin lines with arrows indicate stable and unstable manifolds, homoclinic and heteroclinic connections.

to exist, at least in the vicinity of TB, in saddle-loop bifurcations (SL). At SL, the unstable periodic orbit has grown large and collides with the saddle producing a non-robust homoclinic cycle. The periodic orbit vanishes while the saddle experiences no change whatsoever. The crossing of two bifurcation curves such as SL and $\mathrm{H}$ adds no complexity to the analysis. This is so because whilst $\mathrm{H}$ runs on the node, SL concerns the saddle and, as a consequence, they do not interact. The only effect is to separate regions where the saddle-loop collision occurs in the absence (left of the intersection) or presence (right) of stable modulated waves orbiting the node.

Figure 20 shows phase map diagrams of the flow projected on the two-dimensional centre manifold. The labels indicate to which region in figure 19 each phase map corresponds. Making a clockwise round-trip can help clarify the picture. Starting from (1), where no solutions exist, and crossing $\mathrm{SN}_{+}$into (2) a saddle and a stable node are created. The stable node turns into a stable focus through $\mathrm{NF}_{+}$in (3). In (4), an unstable periodic orbit exists, which has been created in SL. Upon crossing $\mathrm{h}_{+}$into (5), a stable cycle appears around the focus, which becomes unstable. The coexistence of an unstable and a stable cycle in (5) lasts until they collide and disappear in FC, leaving only the saddle and an unstable focus in (7). It is possible to arrive at (7) directly from (4). In this case, the unstable cycle just merges with the stable focus in $\mathrm{h}_{-}$to leave an unstable focus. Crossing $\mathrm{NF}_{-}$, the unstable focus transforms into an unstable node in (8). The unstable focus coalesces with the saddle through $\mathrm{SN}_{-}$back into region (1). The crossing of SL and $\mathrm{h}_{+}$originates a region, (6), where only stable modulated waves exist.

It is at this point necessary to justify why we have not made use of the discrete azimuthal periodicity or of the shift-reflect symmetry in constructing the normal form. The twofold azimuthal periodicity has been enforced in all computations and must therefore be left out of the analysis. We are also entitled to ignore the shiftreflect symmetry because it plays no significant role in the problem at hand, since the flow naturally preserves it. If any of the solutions had broken a symmetry, for which we have found no evidence whatsoever, this would have had to be built into 
the normal form, as symmetry-breaking eigenmodes have the effect of introducing resonances that cancel out some of the normal form coefficients. Since there is no numerical evidence from either time evolution or linear-stability analysis that this happens, these symmetries can be safely ignored.

\section{Conclusion}

A complex bifurcating scenario in pipe Poiseuille flow, involving the appearance of travelling as well as modulated travelling waves, has been thoroughly analysed within a given azimuthal subspace, taking advantage of the special stability properties of the solutions within it. These stability properties make this subspace particularly fit for the search and analysis of all kinds of exotic behaviours including, but not limited to, the double-zero bifurcation unfolded here. This can help place a lower bound to the extreme complexity that must certainly be present in full space pipe flow.

The particular family of waves studied involves the appearance in Hopf bifurcations of modulated waves that are either unstable at onset or become unstable in a fold-ofcycles. Although partial results at a given wavenumber $(\kappa=1.52)$ seem to indicate that the unstable branch of modulated waves collides with lower-branch travelling waves in a saddle-loop bifurcation, it cannot be guaranteed that all unstable modulated waves created in the fold-of-cycles eventually disappear in such a global bifurcation. Their ultimate fate depends on the absence of symmetry-breaking bifurcations that free them from the need of colliding with the saddle or that allow for an entanglement of the stable and unstable manifolds of the saddle in the vicinity of the global bifurcation. While such a behaviour has to be expected generically, the absence of chaotic dynamics around the saddle, as pointed out by random shootings from the lower-branch travelling wave, suggests that this is not the case, and that the scenario described may be fairly accurate.

The Takens-Bogdanov bifurcation experienced by this particular family of travelling waves must not be considered as an exotic scenario. There is strong evidence that other families of travelling waves might be bifurcating in similar environments. This is the case of the highly-symmetric threefold azimuthally-periodic family of travelling waves described by Pringle et al. (2009), for which the eigenvalue associated with the saddle-node bifurcation is known to collide with another eigenvalue and become complex immediately after crossing from lower to upper branch. This happens in the context of instability dictated by several additional unstable eigenvalues, which renders the techniques used in the present study unable to unfold the bifurcation. Nevertheless, a Takens-Bogdanov bifurcation appears to be there all the same. It would not be surprising if this bifurcation scenario were also common in the case of equilibria and relative equilibria identified in other shear flows such as plane Couette or plane Poiseuille, for which a systematic analysis for varying $R e$ and wavenumber is not available in the literature. In these cases, the two continuous wavelengths (spanwise and streamwise) could complicate the picture even further, allowing for codimension-three bifurcations. As a matter of fact, a double-zero bifurcation was identified in the case of Blasius boundary-layer flow (Ehrenstein \& Koch 1995), except that the bifurcation happened to be degenerate in the sense that a $Z_{2}$ symmetry plays an essential role by exactly cancelling the second-order terms of the normal form. The symmetry breaks and the full landscape is substantially altered.

The scenario is nonetheless not general. Other families of travelling waves, such as the mirror-symmetric twofold azimuthally-periodic, do not follow this behaviour. Other travelling waves come from pitchfork bifurcations from other more symmetric 
waves, e.g. the shift-reflect azimuthally non-periodic travelling wave (Pringle \& Kerswell 2007), which comes from symmetry breaking of a mirror-symmetric wave. The Takens-Bogdanov bifurcation simply does not apply in these cases. Therefore, nothing can be said from the results reported about the fate of the branch of modulated waves that arises from a Hopf bifurcation of the shift-reflect non-periodic wave (Duguet et al. 2008a) or of any other periodic solutions that result from scenarios other than a double-zero bifurcation.

As a result of a Takens-Bogdanov bifurcation, no trace of modulated waves is expected to survive long enough to take part in the formation of a chaotic attractor or saddle as a precursor to turbulent dynamics. Nevertheless, it is interesting to point out that, far enough from the Takens-Bogdanov point, only stable upper-branch solutions are left. Thus, all the techniques deployed here may be exploited again for an analysis of the bifurcation cascade they undergo at higher Re. A detailed study of the behaviour of these waves at higher flow rates is currently underway.

This work has been supported by the Spanish Ministry of Science and Technology, under grant FIS2007-61585, by the Catalan Government under grant SGR-00024 and by the Deutsche Forschungsgemeinschaft.

Supplementary movies are available at journals.cambridge.org/flm.

\section{REFERENCES}

BARKLEY, D. 1990 Theory and predictions for finite-amplitude waves in two-dimensional plane Poiseuille flow. Phys. Fluids A 2 (6), 955-970.

Boberg, L. \& Brosa, U. 1988 Onset of turbulence in a pipe. Z. Naturforsch. A: Phys. Sci. 43, 697-726.

Bogdanov, R. 1975 Versal deformations of a singular point on the plane in the case of zero eigenvalues. Funct. Anal. Appl. 9, 144-145.

Brosa, U. \& Grossmann, S. 1999 Minimum description of the onset of pipe turbulence. Eur. Phys. J. B 9 (2), 343-354.

Chossat, P. \& Lauterbach, R. 2000 Methods in Equivariant Bifurcations and Dynamical Systems. World Scientific.

Darbyshire, A. G. \& Mullin, T. 1995 Transition to turbulence in constant-mass-flux pipe flow. J. Fluid Mech. 289, 83-114.

Dennis, J. E. \& Schnabel, R. B. 1996 Numerical Methods for Unconstrained Optimization and Nonlinear Equations. SIAM.

Duguet, Y., Pringle, C. C. T. \& Kerswell, R. R. 2008 a Relative periodic orbits in transitional pipe flow. Phys. Fluids 20 (11), 114102.

Duguet, Y., Willis, A. P. \& Kerswell, R. R. $2008 b$ Transition in pipe flow: the saddle structure on the boundary of turbulence. J. Fluid Mech. 613, 255-274.

Dumortier, F., Roussarie, R., Sotomayor, J. \& Zoladek, H. 1991 Bifurcations of Planar Vector Fields: Nilpotent Singularities and Abelian Integrals. Springer.

ECKHARDT, B. 2009 Introduction. Turbulence transition in pipe flow: 125th anniversary of the publication of Reynolds' paper. Phil. Trans. R. Soc. Lond. A 367, 449-455.

Eckhardt, B., Faisst, H., Schmiegel, A. \& Schneider, T. M. 2008 Dynamical systems and the transition to turbulence in linearly stable shear flows. Phil. Trans. R. Soc. Lond. A 366, $1297-1315$.

Eckhardt, B., Schneider, T. M., Hof, B. \& Westerweel, J. 2007 Turbulence transition in pipe flow. Annu. Rev. Fluid Mech. 39, 447-468.

Ehrenstein, U. \& KoсH, W. 1991 Three-dimensional wave-like equilibrium states in plane Poiseuille flow. J. Fluid Mech. 228, 111-148.

Ehrenstein, U. \& KoсH, W. 1995 Homoclinic bifurcation in Blasius boundary-layer flow. Phys. Fluids 7, 1282-1291.

Faisst, H. \& EcKhardt, B. 2003 Travelling waves in pipe flow. Phys. Rev. Lett. 91 (22), 224502. 
Frayssé, V., Giraud, L., Gratton, S. \& Langou, J. 2003 A set of GMRES routines for real and complex arithmetics on high performance computers. Tech. Rep. TR/PA/03/3. CERFACS. Available at: http://www.cerfacs/algor/Softs.

FreitaG, M. A. 2007 Inner-outer iterative methods for eigenvalue problems: convergence and preconditioning. PhD thesis, University of Bath, Bath, UK.

GASPARD, P. 1990 Measurement of the instability rate of a far-from-equilibrium steady state at an infinite period bifurcation. J. Phys. Chem. 94 (1), 1-3.

Golubitsky, M., LeBlanc, V. G. \& Melbourne, I. 2000 Hopf bifurcation from rotating waves and patterns in physical space. J. Nonlinear Sci. 10 (1), 69-101.

Grossmann, S. 2000 The onset of shear flow turbulence. Rev. Mod. Phys. 72 (2), 603-618.

Guckenheimer, J. \& Holmes, P. 1983 Nonlinear Oscillations, Dynamical Systems, and Bifurcations of Vector Fields. Springer.

Hof, B., van Doorne, C. W. H., Westerweel, J., Nieuwstadt, F. T. M., Faisst, H., Eckhardt, B., Wedin, H., Kerswell, R. R. \& WalefFe, F. 2004 Experimental observation of nonlinear travelling waves in turbulent pipe flow. Science 305 (5690), 1594-1598.

Hof, B., Schneider, T. M., Westerweel, J. \& ECKhardt, B. 2006 Finite lifetime of turbulence in shear flows. Nature 443 (7107), 59-62.

Iooss, G. \& Adelmeyer, M. 1998 Topics in Bifurcation Theory and Applications, 2nd edn. World Scientific.

Kerswell, R. R. \& Tutty, O. R. 2007 Recurrence of travelling waves in transitional pipe flow. J. Fluid Mech. 584, 69-102.

KNOBLOCH, E. 1986 Normal forms for bifurcations at a double zero eigenvalue. Phys. Lett. A 115 (5), 199-201.

KruPA, M. 1990 Bifurcations of relative equilibria. SIAM J. Math. Anal. 21 (6), 1453-1486.

KuZnetsov, Y. A. 1995 Elements of Applied Bifurcation Theory, 3rd edn. Springer.

LehoucQ, R. \& ScotT, J. A. 1996 An evaluation of software for computing eigenvalues of sparse nonsymmetric matrices. Tech. Rep. MCS-P547-1195. Argonne National Laboratory. Available at: http://www.caam.rice.edu/software/ARPACK.

Mamun, C. K. \& Tuckerman, L. S. 1995 Asymmetry and Hopf bifurcation in spherical Couette flow. Phys. Fluids 7 (1), 80-91.

Meerbergen, K., Spence, A. \& Roose, D. 1994 Shift-invert and Cayley transforms for detection of rightmost eigenvalues of nonsymmetric. BIT 34 (3), 409-423.

Mellibovsky, F. \& Meseguer, A. 2009 Cri tical threshold in pipe flow transition. Phil. Trans. $R$. Soc. Lond. A 367, 545-560.

Meseguer, A., Avila, M., Mellibovsky, F. \& Marques, P. 2007 Solenoidal spectral formulations for the computation of secondary flows in cylindrical and annular geometries. Eur. Phys. J. Special Topics 146, 249-259.

Meseguer, A. \& Mellibovsky, F. 2007 On a solenoidal Fourier-Chebyshev spectral method for stability analysis of the Hagen-Poiseuille flow. Appl. Numer. Maths 57, 920-938.

Meseguer, A. \& Trefethen, L. N. 2003 Linearized pipe flow to Reynolds number 10 ${ }^{7}$. J. Comput. Phys. 186, 178-197.

Nagata, M. 1997 Three-dimensional traveling-wave solutions in plane Couette flow. Phys. Rev. E 55 (2), 2023-2025.

Pfenniger, W. 1961 Transition in the inlet length of tubes at high Reynolds numbers. In Boundary Layer and Flow Control (ed. G. V. Lachman), pp. 970-980. Pergamon.

Pringle, C. C. T., Duguet, Y. \& Kerswell, R. R. 2009 Highly symmetric travelling waves in pipe flow. Phil. Trans. R. Soc. Lond. A 367, 457-472.

Pringle, C. C. T. \& Kerswell, R. R. 2007 Asymmetric, helical, and mirror-symmetric traveling waves in pipe flow. Phys. Rev. Lett. 99 (7), 074502.

Pugh, J. D. \& Saffman, P. G. 1988 Two-dimensional superharmonic stability of finite-amplitude waves in plane Poiseuille flow. J. Fluid Mech. 194, 295-307.

Quarteroni, A., SACCO, R. \& SAleri, F. 2007 Numerical Mathematics, 2nd edn. Springer.

RAND, D. 1982 Dynamics and symmetry: predictions for modulated waves in rotating fluids. Arch. Rat. Mech. Anal. 79 (1), 1-37.

REYNOLDS, O. 1883 An experimental investigation of the circumstances which determine whether the motion of water shall be direct or sinuous and of the law of resistance in parallel channels. Phil. Trans. R. Soc. Lond. 174, 935-982. 
Sanchez, J., Marques, F. \& Lopez, J. M. 2002 A continuation and bifurcation technique for Navier-Stokes flows. J. Comput. Phys. 180 (1), 78-98.

Schmid, P. J. \& Henningson, D. S. 1994 Optimal energy growth in Hagen-Poiseuille flow. J. Fluid Mech. 277, 197-225.

Schneider, T. M., EcKhardt, B. \& Vollmer, J. 2007 a Statistical analysis of coherent structures in transitional pipe flow. Phys. Rev. E 75 (6), 066313.

Schneider, T. M., EcKhardt, B. \& Yorke, J. A. $2007 \mathrm{~b}$ Turbulence transition and edge of chaos in pipe flow. Phys. Rev. Lett. 99 (3), 034502.

Shan, H., Ma, B., Zhang, Z. \& Nieuwstadt, F. T. M. 1999 On the spatial evolution of a wall-imposed periodic disturbance in pipe Poiseuille flow at $R e=3000$. Part 1. Subcritical disturbance. J. Fluid Mech. 398, 181-224.

Skufca, J. D., Yorke, J. A. \& Eckhardt, B. 2006 Edge of chaos in a parallel shear flow. Phys. Rev. Lett. 96 (17), 174101.

Smale, S. 1967 Differentiable dynamical systems. I. Diffeomorphisms. Bull. Am. Math. Soc. 73 (6), 747-817.

Solbelman, I. \& Meiron, D. I. 1991 Finite-amplitude bifurcations in plane Poiseuille flow: twodimensional Hopf-bifurcation. J. Fluid Mech. 229, 389-416.

Takens, F. 1974 Singularities of vector fields. Publ. Math. IHES 43, 47-100.

VIswanath, D. 2007 Recurring motions within plane Couette turbulence. J. Fluid Mech. 580, 339358.

WALEFFE, F. 1995 Hydrodynamic stability and turbulence: beyond transients to a self-sustaining process. Stud. Appl. Maths 95 (3), 319-343.

Waleffe, F. 1997 On a self-sustaining process in shear flows. Phys. Fluids 9 (4), 883-900.

WANG, J., Gibson, J. \& WalefFe, F. 2007 Lower branch coherent states in shear flows: transition and control. Phys. Rev. Lett. 98 (20), 204501.

Wedin, H. \& Kerswell, R. R. 2004 Exact coherent structures in pipe flow: travelling wave solutions. J. Fluid Mech. 508, 333-371.

Wiggins, S. 2003 Introduction to Applied Nonlinear Dynamical Systems and Chaos, 2nd edn. Springer.

Willis, A. P. \& Kerswell, R. R. 2008 Coherent structures in localized and global pipe turbulence. Phys. Rev. Lett. 100 (12).

Wygnanski, I. J. \& Champagne, F. H. 1973 On transition in a pipe. Part 1. The origin of puffs and slugs and the flow in a turbulent slug. J. Fluid Mech. 59, 281-335.

Wygnanski, I. J., Sokolov, M. \& Friedman, D. 1975 On transition in a pipe. Part 2. The equilibrium puff. J. Fluid Mech. 69, 283-304.

Zikanov, O. Y. 1996 On the instability of pipe Poiseuille flow. Phys. Fluids 8 (11), 2923-2932. 